\title{
A systematic review of nutritional risk factors of Parkinson's disease
}

\author{
Lianna Ishihara* and Carol Brayne \\ Department of Public Health and Primary Care, University of Cambridge, Forvie Site, Robinson Way, \\ Cambridge CB2 2SR, UK
}

\begin{abstract}
A wide variety of nutritional exposures have been proposed as possible risk factors for Parkinson's disease (PD) with plausible biological hypotheses. Many studies have explored these hypotheses, but as yet no comprehensive systematic review of the literature has been available. MEDLINE, EMBASE, and WEB OF SCIENCE databases were searched for existing systematic reviews or meta-analyses of nutrition and $\mathrm{PD}$, and one meta-analysis of coffee drinking and one meta-analysis of antioxidants were identified. The databases were searched for primary research articles, and articles without robust methodology were excluded by specified criteria. Seven cohort studies and thirty-three case-control (CC) studies are included in the present systematic review. The majority of studies did not find significant associations between nutritional factors and PD. Coffee drinking and alcohol intake were the only exposures with a relatively large number of studies, and meta-analyses of each supported inverse associations with PD. Factors that were reported by at least one CC study to have significantly increased consumption among cases compared with controls were: vegetables, lutein, xanthophylls, xanthins, carbohydrates, monosaccharides, junk food, refined sugar, lactose, animal fat, total fat, nuts and seeds, tea, Fe, and total energy. Factors consumed significantly less often among cases were: fish, egg, potatoes, bread, alcohol, coffee, tea, niacin, pantothenic acid, folate and pyridoxine. In three cohort studies, two reported borderline decreased relative risks and one a significant increased risk with vitamin $\mathrm{C}$ intake. One cohort reported an inverse association between caffeine intake and PD. Three cohorts reported significant positive association in men between dairy products and PD.
\end{abstract}

Parkinson's disease: Nutrition: Systematic review: Antioxidant risk

\section{Introduction}

The specific contributions of genetic and environmental factors to the aetiology of Parkinson's disease (PD) remain largely unknown, nearly 200 years after PD was first recognised by James Parkinson (Parkinson, 1817). As the elderly proportion of the world's population increases, questions surrounding ageing disorders become increasingly pertinent. PD is the second most common neurological disorder and its worldwide prevalence is estimated to be approximately $2 \%$ in those over 65 years (Zhang \& Roman, 1993; de Rijk et al. 1997c).

Concordance rates from monozygotic and dizygotic twin studies are similarly low, which indicates that genetics cannot fully explain the aetiology of PD (Tanner, 2003). Specific causal genes have been identified in familial PD, but these genes are relatively rare in sporadic PD (de Silva et al. 2000; Gwinn-Hardy, 2002). Therefore it is probable that environmental factors contribute to the aetiology.

The possible role of nutrition in the aetiology of PD has been examined in many epidemiological studies; however, the implicated foods and nutrients vary between studies. Most have been retrospective case-control (CC) studies; therefore exposure measurement may not reflect the premorbid diet. Many were exploratory, testing multiple nutritional risk factors with the purpose of generating hypotheses.

Specific foods, macronutrients and micronutrients could contribute to the cause of PD either by direct or indirect effects, as with amyotrophic lateral sclerosis-Parkinsonism dementia complex, which is caused by neurotoxic cycad seeds (Calne et al. 1986; Cox \& Sacks, 2002). This exposure is uncommon in other parts of the world, but it does highlight food consumption as a possible risk factor for PD.

Nutrition may influence neurodegeneration by indirect effects on the body. The most relevant biological mechanism for the role of nutrition in PD is oxidative stress, which is defined as increasing production of reactive oxygen or nitrogen species and/or decreasing levels of antioxidant defences (Foley \& Riederer, 2000; Bharath et al. 2002; Butterfield et al. 2002; Koutsilieri et al. 2002; Rao \& Balachandran, 2002; Jenner, 2003). There is an ongoing

\footnotetext{
Abbreviations: CC, case-control; HPFS, Health Professionals' Follow-up Study; NCC, nested case-control; NHS, Nurses' Health Study; OR, odds ratio; PD, Parkinson's disease; RR, relative risk.

* Corresponding author: Lianna Ishihara, fax +44 1223 330330, email Lsi20@medschl.cam.ac.uk
} 
debate as to whether oxidative stress is a cause or consequence of neurodegeneration. The brain is susceptible to oxidative damage because of its high $\mathrm{O}_{2}$ utilisation, high $\mathrm{Fe}$ content and the presence of excess unsaturated fatty acids, which are targets for lipid peroxidation (Halliwell, 1992; Bharath et al. 2002).

Preliminary evidence that alcohol may contribute to oxidative stress through production of alkaloids, which may be toxic, is inconclusive (Collins, 2002). If this is the case, then there should be an increased risk of PD in individuals who have ever consumed alcohol, and a dose-response effect.

A systematic search of the published literature for all studies reporting on the association between nutritional risk factors and PD has been conducted. To date there have not been any comprehensive systematic reviews on this topic. The evidence for the association of PD with various foods and nutrients is presented.

\section{Methods \\ Search strategy}

To determine if previous systematic reviews or metaanalyses of nutritional risk factors for PD existed, a search for all reviews of PD and risk factors was conducted using MEDLINE, EMBASE and WEB OF SCIENCE databases from 1989 through to September 2004. Systematic reviews or meta-analyses were assessed on their search methodology, inclusion criteria, and presentation of results. If the review met the assessment criteria, then review results were reported along with additional relevant results.

To identify original contributions to the topic, the same three databases were searched based on published sources (Oxman, 1994; Glasziou et al. 2001; Scottish Intercollegiate Guidelines Network, 2002; Khan, 2003), and by using index terms from databases within MEDLINE and EMBASE. The MEDLINE search used the following medical subject headings terms and free text with ' $*$ ' as the wildcard symbol: Parkinson's disease and one or more of the following (nutrition; diet*; food; food additives; food, fortified; ascorbic acid; vitamin; antioxidant*; antioxidants; oxidat*; iron; mineral; metal*; fat*; caffeine; alcohol; beverage*). EMBASE was searched for terms similar to MEDLINE using EMBASE index terms. WEB OF SCIENCE did not have an index of terms; therefore terms from MEDLINE and EMBASE were used.

Bibliographies from the primary search and reviews were scanned and additional relevant references were obtained. No attempt was made to solicit unpublished results, either directly from key investigators or through conference abstracts.

\section{Inclusion and exclusion criteria}

Inclusion: (1) articles published in all languages; (2) primary research that examined the relationship between specific foods, food groups or nutrients and PD; (3) PD as the outcome of interest; (4) reported odds ratios (OR) or relative risks (RR) and $95 \% \mathrm{CI}$, or provided enough information to calculate values; (5) 'ever v. never', semi- quantitative or quantitative measures of exposure; (6) an attempt to assess the exposure information before the onset of disease.

Exclusion: (1) cross-sectional studies without CC design; (2) for articles that presented the same data in multiple publications, all but the most recent publication were excluded; (3) used a subjective measure of exposure (for example, of a husband and wife pair, which ate more than the other).

Studies without either specified diagnostic criteria, in medical records or upon examination by a neurologist, or a physician-verified diagnosis were excluded. Ecological studies are not included because exposure is not necessarily related to disease in the same individuals. The present review will specifically look at associations between nutrition and PD in man. An extensive literature base in animals does exist.

\section{Data extraction}

The Newcastle-Ottawa scale and other guidelines for assessing the quality of non-randomised studies in metaanalyses were used to identify study characteristics for data extraction (Liddle, 1996; Glasziou et al. 2001; Wells et al. 2003). Subjective quality scoring was not conducted. One author (L. I.) extracted data from studies. Only objective study characteristics that could contribute to heterogeneity in results were extracted and summarised in tables.

\section{Summary tables}

Figures for alcohol and an overall summary table are presented. Tables for other food groups are available online at http://www.iph.cam.ac.uk/ 1si20/NRR_tables.htm

The tables include the most adjusted OR and RR estimates from each study. Factors are organised by categories within each broad classification of exposure (for example, carbohydrates and fats). CC studies are presented first, followed by nested $\mathrm{CC}$ (NCC) and cohort studies, each in the order of ascending publication date.

The adjustment column includes the matching variables, if used in the analysis, and adjustments made during the analysis. The number of cases and controls, or the cohort size, are reported.

\section{Calculation of missing odds ratios and confidence intervals}

If the OR and/or $95 \% \mathrm{CI}$ were not presented in the original study, the values were calculated from available information. Studies without sufficient information to calculate both the OR and $95 \%$ CI were not included in the present review.

The STATA statistical package (StataCorp LP, College Station, TX, USA) was used. The crude OR and $95 \%$ CI for CC studies were calculated with the STATA cci command. The STATA mcci command was used for matched CC studies.

For coffee and alcohol, when possible, the quantitative measures were also calculated as OR or RR, yes v. no, to increase the comparability of estimates between studies. The CC OR were calculated as described above. The cohort 
RR and $95 \%$ CI were calculated using the STATA iri function.

\section{Meta-analysis}

A pooled risk estimate was calculated only for variables investigated by more than five studies with homogeneous exposure measurement. This was decided after data extraction was completed. The STATA meta command was used to produce random and fixed effect estimates and to conduct a test of heterogeneity.

\section{Results}

\section{Literature search}

The titles and abstracts of possible systematic reviews and meta-analyses were reviewed, and one meta-analysis each for coffee drinking and antioxidants were identified (Hernan et al. 2002; Etminan et al. 2005).

After applying inclusion and exclusion criteria, there were a total of seven cohorts and thirty-three CC studies included in the systematic review from primary and secondary searches. All primary articles were found in either MEDLINE or EMBASE. No unique articles were identified through WEB OF SCIENCE. Additional studies were added after searching the bibliographies of primary articles and reviewing other environmental risk factor publications.

One cross-sectional study is included because the methods were similar to the CC studies, and because it was included in one of the published meta-analyses (de Rijk et al. 1997a). The quality of evidence is not as strong, but results are presented as supporting evidence.

\section{Study characteristics and methods}

Cohort study populations. There were six prospective cohorts from the USA and one cohort from the Netherlands (Table 1). There were two prospective NCC studies, one from the Honolulu Heart Study cohort (Morens et al. 1996; Paganini-Hill, 2001). The Health Professionals' Follow-up Study (HPFS) and the Nurses' Health Study (NHS) recruited participants in healthcare occupations through a mailed survey. Both are selective populations and may not be representative of the general population, but they had relatively good response and follow-up rates. The Honolulu Heart Study is a male-only selective population originally chosen to study heart disease among Japanese immigrants in Hawaii. It has since expanded to look at other outcomes including age-related diseases, and is now known as the Honolulu Aging Study. The Iowa cohort of women did not describe methods for recruitment. The remaining cohorts, Rotterdam, Framingham and Leisure World, were community based.

Case-control selection. The methods for case and control selection were heterogeneous (Table 2). Cases were selected from hospitals, neurology clinics, patient support groups, and pharmacy databases. The source of cases was most often neurology and movement disorders clinics, which may

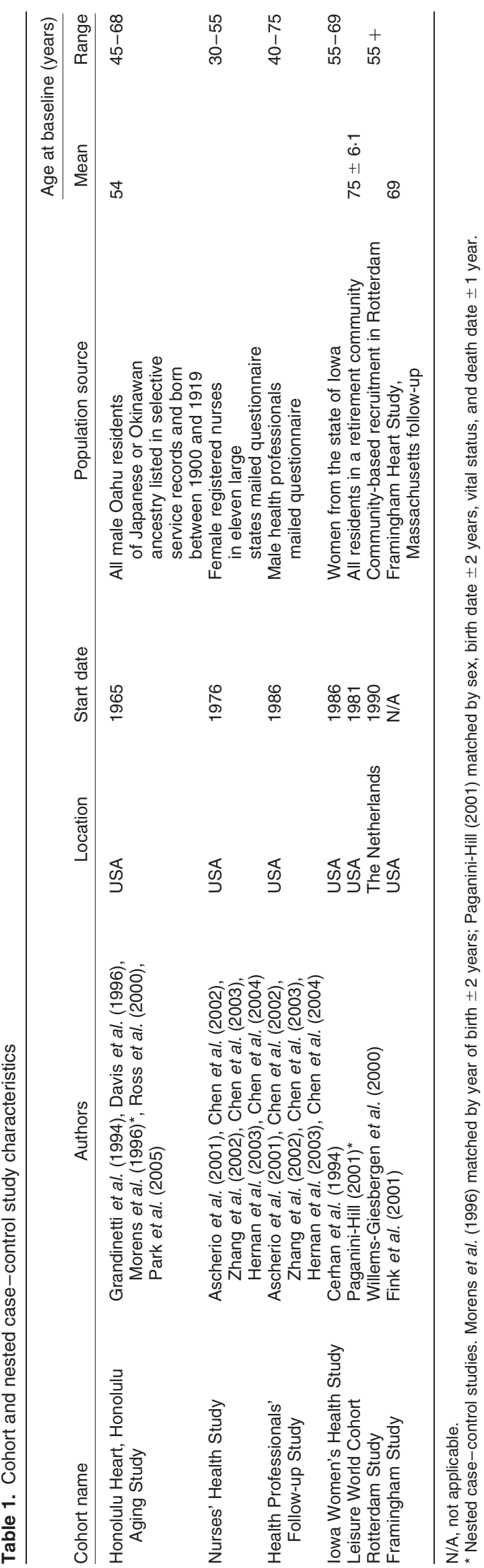




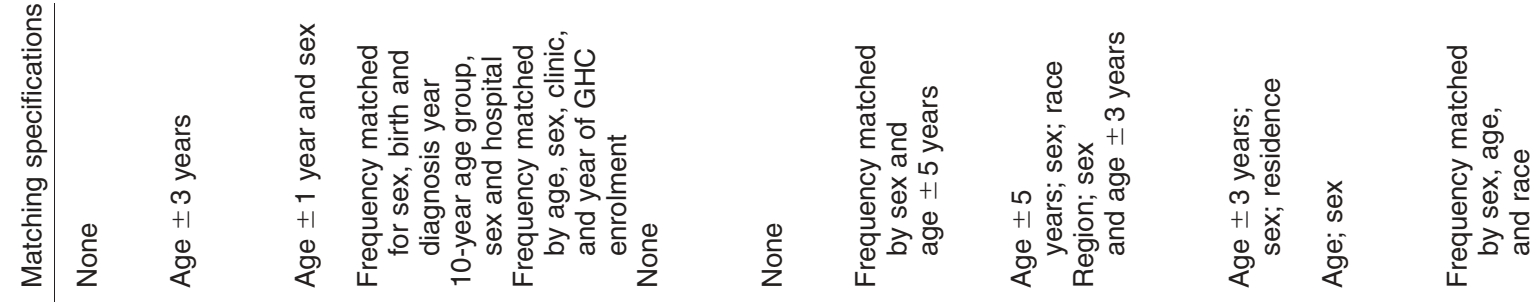

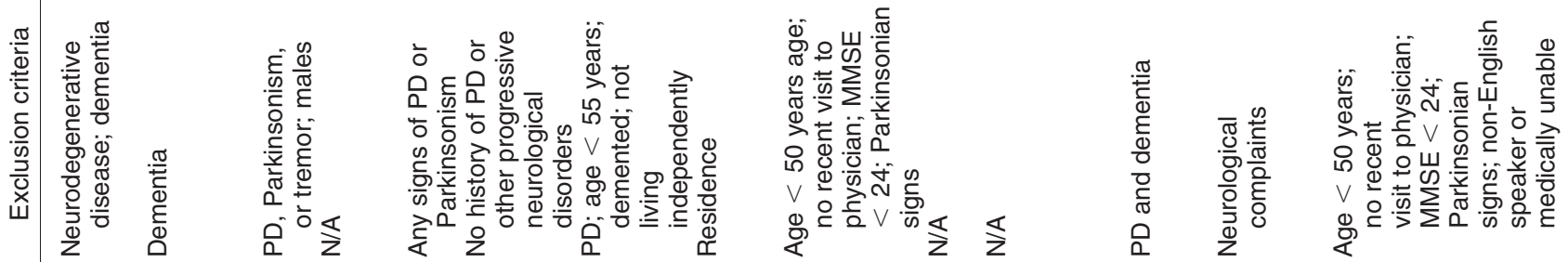

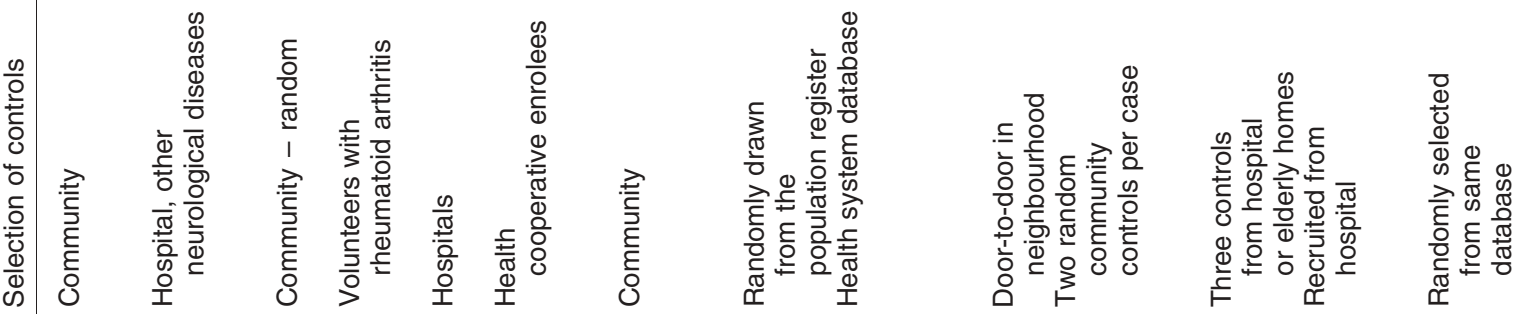

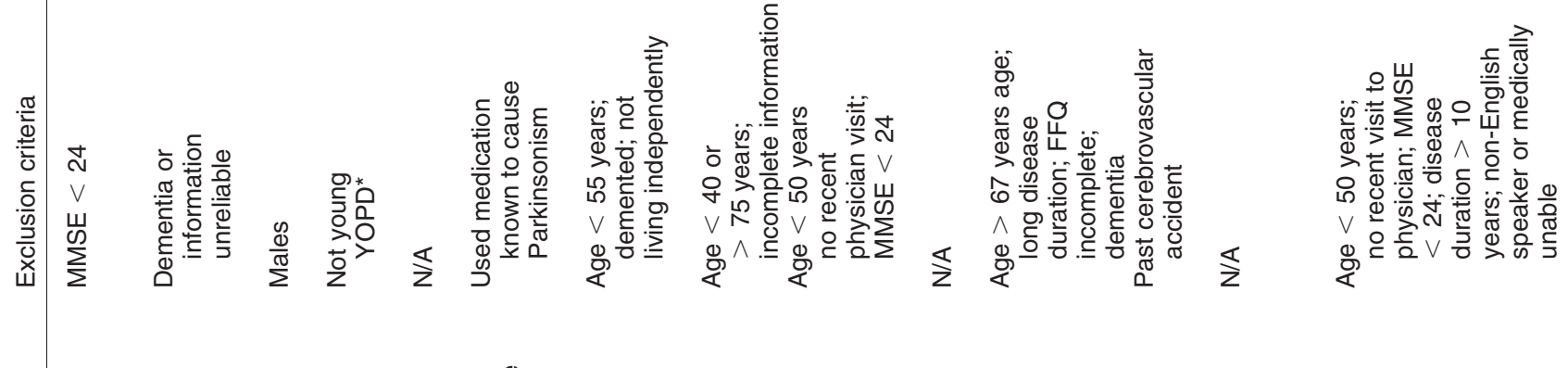

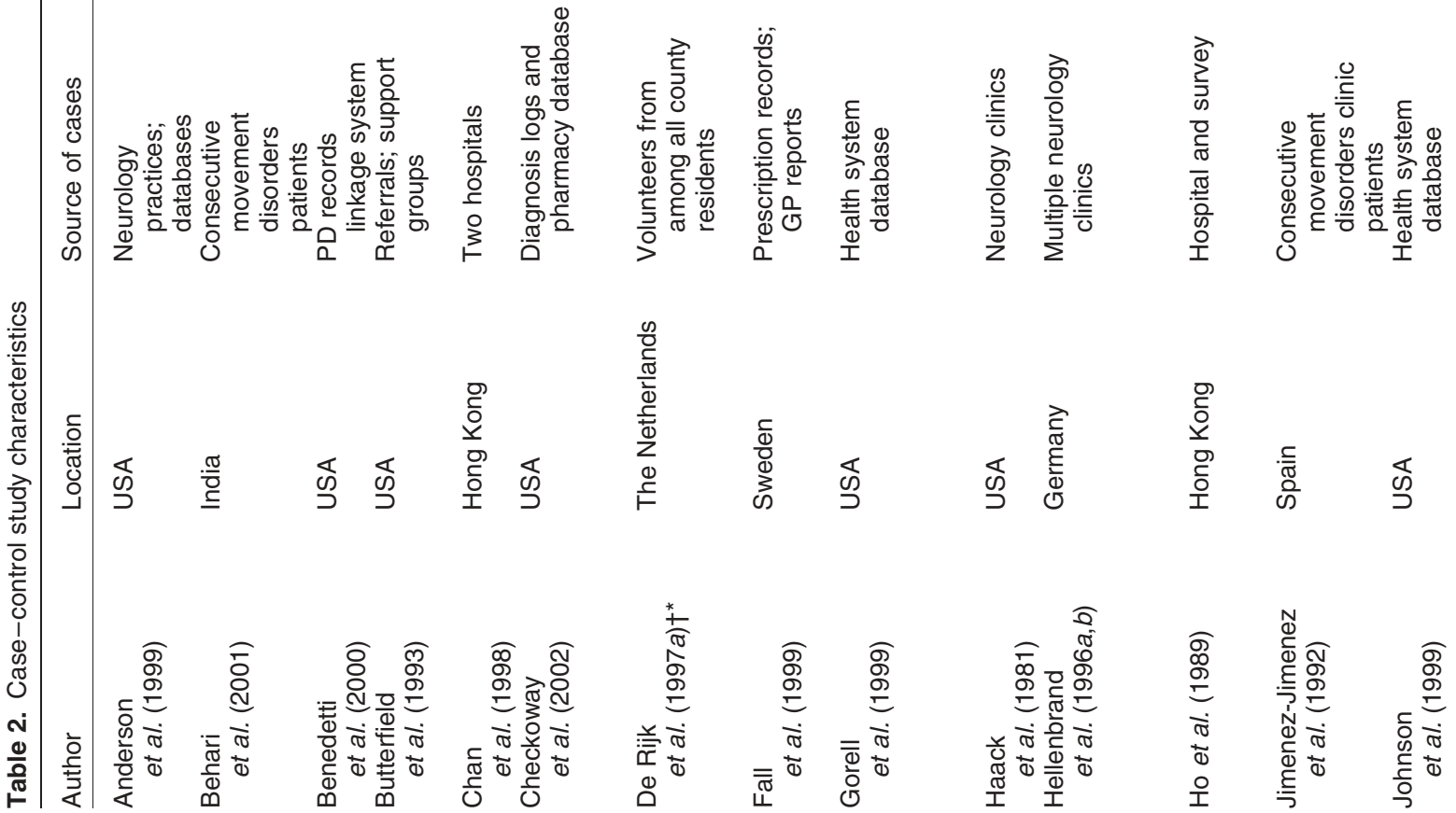




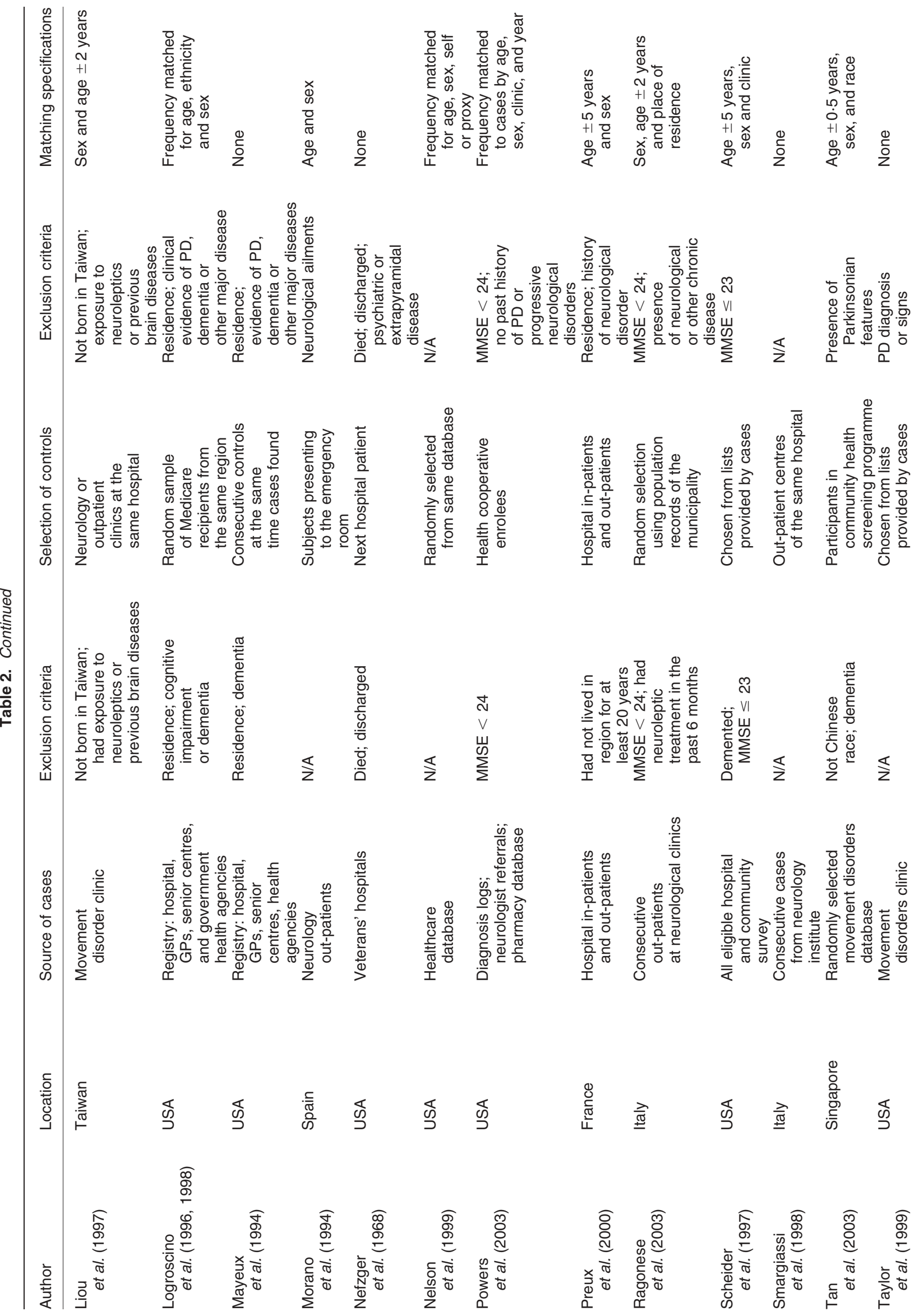




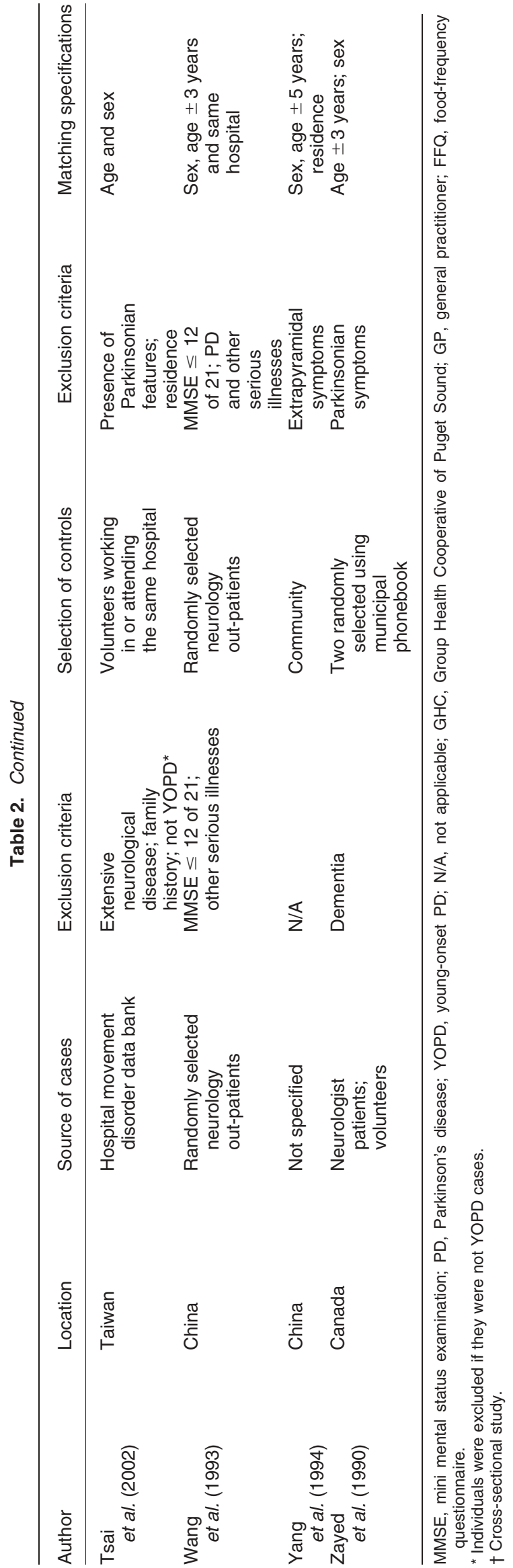

be a selected group of PD patients. Controls were selected from among spouses, the patients' communities, hospitals, and healthcare databases. Most of the studies matched controls to the cases by age and sex, either directly or by frequency matching, with the exception of six (Nefzger et al. 1968; Mayeux et al. 1994; Smargiassi et al. 1998; Anderson et al. 1999; Fall et al. 1999; Taylor et al. 1999). Other matching criteria included ethnicity, hospital or clinic, and geographical region.

A common exclusion criterion was dementia, usually indicated by a mini mental status examination (Folstein et al. 1975) score below twenty-four, because subjects with dementia would be unable to provide reliable information. Other exclusion criteria related to age, disease duration, practical considerations such as location and language, and medical history, especially diseases that may have a role in PD aetiology.

Exposure measurement. Food and nutrient intake can be assessed using different instruments, including the $24 \mathrm{~h}$ dietary recall and semi-quantitative food-frequency questionnaire. Food-frequency questionnaires and study-specific questionnaires were the most commonly used instruments in CC and cohort studies (Table 3).

Exposure measurement may be qualitative, semiquantitative, or quantitative. The simplest qualitative measure of consumption is the 'ever $v$. never' or 'yes $v$. no' question. Another qualitative measure is a relative measure, such as asking whether the case ate more or less of a food item than his/her spouse before PD onset. The results from relative comparisons are excluded because they do not provide estimates of consumption differences between cases and controls.

A semi-quantitative measurement asks about the average or usual number of times a food is consumed during a given time period. The approximate serving size may be specified.

Quantitative measurements are more precise about serving sizes and frequency of intake. The subjects may be given graphs with serving sizes to estimate the amounts of food. A more precise measure called 'weighed intake' requires subjects to weigh all foods consumed. This is not economical and is rarely seen in epidemiological studies, except as a means of validation on a sub-sample of the study population.

Risk estimates were calculated for semi-quantitative and quantitative measures as: above $v$. below the median; highest $v$. lowest tertiles, quartiles, and quintiles; number of servings or times consumed in a given time period; continuous measures using logistic regression.

Diagnostic criteria. The diagnosis of Parkinsonism is based on clinical signs because there is currently no conclusive diagnostic test available (Tanner \& Ben Shlomo, 1999). The gold standard is a post-mortem pathological examination. PD clinical characteristics manifest only when the substantia nigral cell loss has reached a threshold of 60 to $80 \%$, and most Parkinsonian symptoms are not specific to PD (Lang \& Lozano, 1998; Ben-Shlomo, 1998; Tanner \& Ben Shlomo, 1999). PD is underdiagnosed in the population because of mis- and late diagnosis (Hughes et al. 1992b; Ben-Shlomo, 1998). 


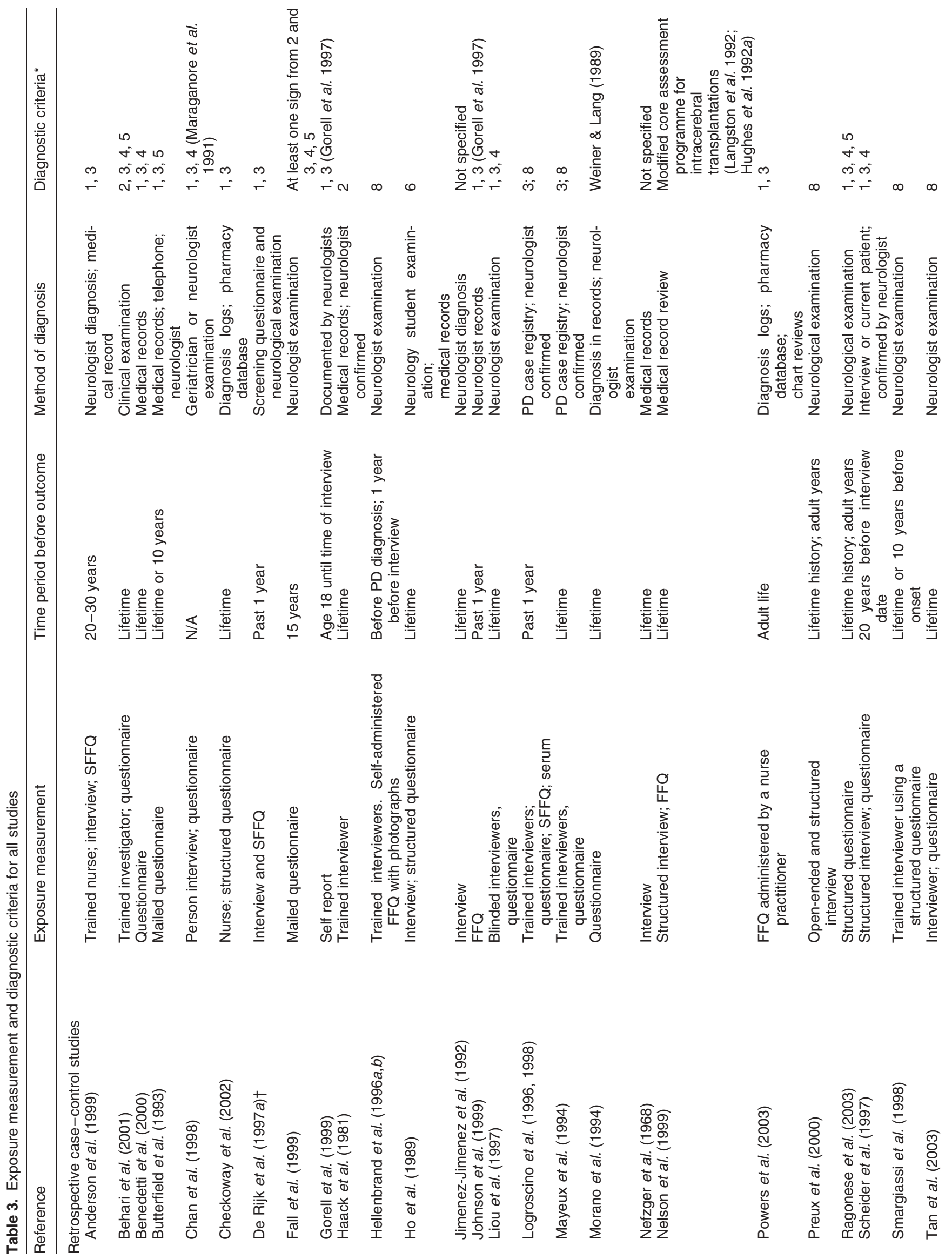




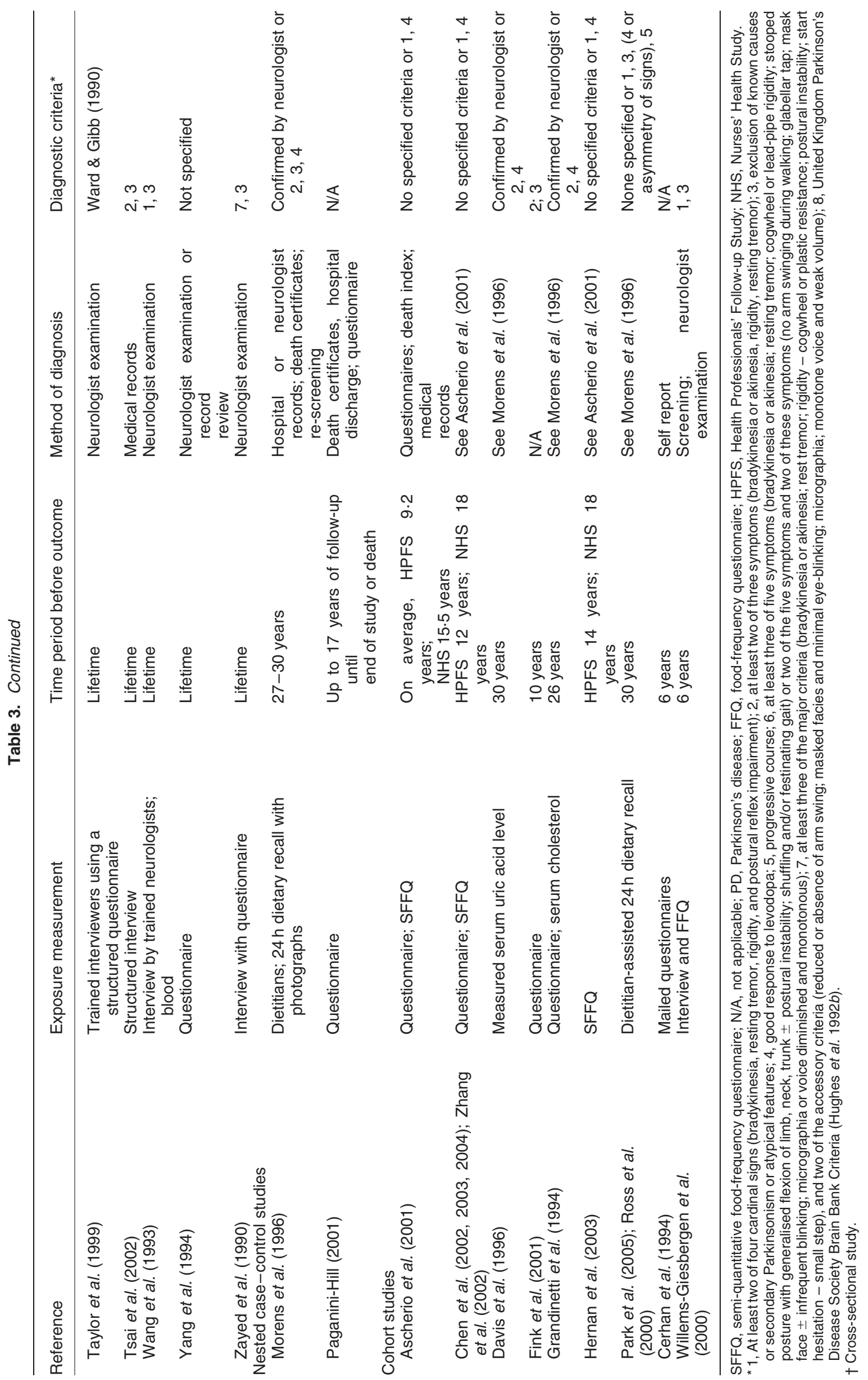


Methods and criteria can significantly impact PD diagnosis (de Rijk et al. 1997b). Both varied between the reviewed studies and their characteristics are reported as possible sources of heterogeneity that could influence risk estimates (Table 3).

Methods of diagnosis used in the studies included medical record review, interview, physician examination and neurological examination. Death certificates were not considered an acceptable method of PD case ascertainment because PD is often not the primary cause of death. Medical record review and interview only identify previously diagnosed cases. Clinical or neurological examinations can potentially identify new cases of PD and exclude secondary causes of PD. The misdiagnosis rate of PD in a clinicopathological study was $24 \%$ by neurologists; therefore it is possible that non-neurologically trained clinicians would have a higher misdiagnosis rate (Hughes et al. 1992b).

Diagnostic criteria are often based upon the presence of four cardinal signs of Parkinsonism: bradykinesia or akinesia, resting tremor, rigidity, and postural reflex impairment. Secondary Parkinsonism, caused by known sources such as neuroleptic drugs or 1-methyl-4-phenyl1,2,3,6-tetrahydropyridine exposure, should be excluded. Exclusion criteria based on secondary Parkinsonism and known causes were specified by studies; however, they are not undisputed. For example, stroke was excluded as a known cause in some studies, but examined as a risk factor in others. Levodopa response is often used as supporting evidence for PD although not all patients take this drug. Other features of Parkinsonism such as micrographia, unilateral onset, progressive course, shuffling gait, reduced arm swing and masked facies may be used as supporting evidence.

The reviewed studies used a combination of the symptoms and supporting features mentioned above to diagnose PD (Table 3). Some studies did not specify diagnostic criteria and accepted the medical diagnosis by a neurologist or other clinician. The most commonly used set of criteria was from the United Kingdom Parkinson's Disease Society Brain Bank (Hughes et al. 1992a,b) (Appendix 1).

\section{Fruits, vegetables and antioxidants}

Fruits and vegetables have relatively high contents of nutrients such as vitamin $C$, vitamin $A$, and $\beta$-carotene. They were used in previous studies to approximate antioxidant intake. Higher intake of fruits and vegetables is hypothesised to be protective for PD because of the nutrient-rich content, but there is also the possibility that the use of pesticides on raw produce may increase risk (Ho et al. 1989).

Intake was measured as: fruits, vegetables, combined fruits and vegetables, raw vegetables, and tomatoes only. Exposure was reported as 'yes or no' (Ho et al. 1989), consumption less than or greater than or equal to one time per week (Chan et al. 1998), and quartiles of consumption (Hellenbrand et al. 1996b; Anderson et al. 1999; Chen et al. 2002). For tomatoes, intake was recorded as seldom/never, each month, each week or daily (Fall et al. 1999).
There were no clear differences between $\mathrm{CC}$ and cohort risk estimates for fruits and vegetables. The only significantly increased OR was found for raw vegetables by the CC study with the smallest sample size (Ho et al. 1989). The study was conducted in Hong Kong, as was a larger study by Chan et al. (1998), which reported a nonsignificant increased OR for raw vegetable consumption. The German study (Hellenbrand et al. 1996b) found a nonsignificant inverse association. The exposure was binary compared with the two CC studies with null findings, which used quantitative measures. Hellenbrand reported OR by quartiles and Chan by greater or less than one serving per $\mathrm{d}$. Studies were adjusted for age, except for the two studies from Hong Kong. The NHS and HPFS cohorts reported sexspecific risk estimates, which suggested that men but not women have a lower risk of PD with higher fruit and vegetable intake, although the results were not significant (Chen et al. 2002).

Vitamin A and carotenoids. Vitamin A and carotenoids have been shown to have antioxidant properties in animal studies but their effects in man have not been consistently demonstrated (Institute of Medicine, 2001; US Department of Agriculture, 2004). $\beta$-Carotene is a provitamin A carotenoid. Lycopene, lutein, zeaxanthin, and xanthophyll are carotenoids, but they are not converted to vitamin A.

Vitamin A exposure was measured as: consumption of foods high in vitamin A, retinol, dietary and total intake. The exposure was reported in quartiles (Anderson et al. 1999; Johnson et al. 1999) or tertiles (Cerhan et al. 1994; Paganini-Hill, 2001). The two CC studies did not find an association between vitamin A intake and PD; however, Anderson reported the only estimate below unity for foods containing vitamin A (Anderson et al. 1999; Johnson et al. 1999). The female cohort (Cerhan et al. 1994) and NCC studies (Paganini-Hill, 2001) reported borderline significant increased risks for total vitamin A intake. In these two studies, the risk estimates of the sub-categories dietary vitamin A (Paganini-Hill, 2001) and retinol (Cerhan et al. 1994) were calculated, and they remained elevated but not significant.

Other antioxidant exposures included total antioxidants, $\beta$-carotene, $\alpha$-carotene, total carotenes, lutein, lutein and zeaxanthin, lycopene and other xanthins. Intake was classified by quartiles (Hellenbrand et al. 1996a; Anderson et al. 1999; Johnson et al. 1999; Powers et al. 2003), quintiles (Zhang et al. 2002), median intake (Scheider et al. 1997), or specified amounts of intake (de Rijk et al. 1997a).

There was no association found between total antioxidant intake and $\mathrm{PD}$ in one CC study comparing highest and lowest quartiles (Anderson et al. 1999). There was no significant association between PD and flavonoid intake reported by one cross-sectional study (de Rijk et al. 1997a).

The cross-sectional study reported a non-significant inverse association for $\beta$-carotene. A meta-analysis (Etminan et al. 2005) for $\beta$-carotene intake reported nonsignificant inverse pooled estimates for one cross-sectional (de Rijk et al. 1997a) and three CC studies (Hellenbrand et al. 1996a; Scheider et al. 1997; Johnson et al. 1999).

Only one CC study measured total carotenes, above and below the median, and reported a non-significant positive 
association (Scheider et al. 1997). Results for $\alpha$-carotene were not significant. The cohort study (Zhang et al. 2002) reported an inverse relationship with PD, and the CC study (Scheider et al. 1997) found a positive association with $\alpha$ carotene intake. Both studies adjusted for age, sex, smoking and energy intake.

Two CC studies and one cohort study reported nonsignificant associations for lycopene. The larger CC study found an increased OR (Powers et al. 2003). The cohort study reported a decreased RR (Zhang et al. 2002).

Three CC studies found positive associations between PD and lutein, and two were significant (Scheider et al. 1997; Johnson et al. 1999; Powers et al. 2003). The OR decrease with more recent publication date, and the non-significant estimate comes from the largest study. All were adjusted for age and sex. The only cohort study reported a nonsignificant decreased risk for intake of lutein and zeaxanthin combined (Zhang et al. 2002).

Total xanthophylls and other xanthins showed a significant positive association with $\mathrm{PD}$, comparing above and below the median in one CC study (Scheider et al. 1997). The same study found a non-significant increased risk among those consuming high amounts of cryptoxanthin. A cohort study found a nearly significant inverse association between $\beta$-cryptoxanthin and PD (Zhang et al. 2002).

In summary, there were no significant associations reported between vitamin A intake and PD for CC studies but an NCC study and a female cohort study reported borderline significant increased RR. Alpha-, $\beta$ - and total carotenes were not associated with PD. Lycopene had a borderline significant increased OR in one CC study, but no association in one cohort and one CC study. Higher intakes of lutein were associated with PD in CC studies, but not in two cohorts. Only one study examined other carotenerelated nutrients, and significantly increased OR were found for xanthins and xanthophylls, but not for cryptoxanthin and $\beta$-cryptoxanthin.

Vitamin C. Vitamin C (ascorbic acid) has antioxidant properties (Ensminger, 1994; US Department of Agriculture, 2004). Vitamin C intake was assessed by: intake of foods containing vitamin $\mathrm{C}$, total intake, dietary intake and use of supplements. The risks are reported for quintiles (Zhang et al. 2002), quartiles (Hellenbrand et al. 1996a; Anderson et al. 1999; Johnson et al. 1999; Powers et al. 2003), tertiles (Cerhan et al. 1994; Paganini-Hill, 2001) and median intake (Scheider et al. 1997).

There were no significant associations between PD and vitamin $\mathrm{C}$, with the exception of the NCC. The crosssectional study reported no association between vitamin $\mathrm{C}$ per $1000 \mathrm{mg} / \mathrm{d}$ and PD. One CC study found a decreased OR for higher consumption of foods with vitamin $\mathrm{C}$ (Anderson et al. 1999). Of four CC studies, two reported an increased risk and two a decreased risk with higher intake of vitamin C. The highest estimate was from the smallest study, and the estimates decreased with increasing sample size. All studies were adjusted for age and sex. There were no clear differences between the adjustment factors for studies reporting opposing estimates. The estimates did not follow a trend for date of publication.
In the cohort studies, men had an increased risk of PD and women a decreased risk with increasing vitamin $\mathrm{C}$ intake (Zhang et al. 2002). The other female cohort reported a decreased risk for total vitamin $\mathrm{C}$ intake (Cerhan et al. 1994).

Dietary intake was examined as a sub-category of total vitamin $\mathrm{C}$ intake by the NCC study and the NHS and HPFS cohorts. The NCC study reported a significantly increased risk of PD. The cohort studies reported decreased risks for men and women, separately and combined. All adjusted for age and sex, and the cohort estimates were further adjusted.

A meta-analysis (Etminan et al. 2005) of dietary vitamin $\mathrm{C}$ intake reported non-significant inverse pooled estimates for five CC (Hellenbrand et al. 1996a; Scheider et al. 1997; Anderson et al. 1999; Johnson et al. 1999; Paganini-Hill, 2001), one cohort (Zhang et al. 2002), and one crosssectional study (de Rijk et al. 1997a).

The NHS and HPFS cohorts examined the association between vitamin $\mathrm{C}$ supplement use and PD (Zhang et al. 2002). There were no significant RR when comparing past use with never in men. However, for women there was a borderline increased risk of PD. In contrast, when current use for 10 years or more was compared with never users, men had an increased risk and women a decreased risk, although both were not significant. Comparing highest with lowest quintiles of vitamin $\mathrm{C}$ supplements, men had a slightly increased risk and women a decreased risk, although neither was significant.

Overall, there was no consistent evidence for an association between vitamin $\mathrm{C}$ intake and PD. The borderline significant estimates were in opposition. The Iowa women's cohort reported a decreased risk of PD with increased vitamin $\mathrm{C}$ intake, whereas the NCC study reported a significantly increased risk for dietary intake. The NHS women's cohort found an increased risk of PD with past use of vitamin $\mathrm{C}$ supplements compared with never use.

Vitamin E. $\alpha$-Tocopherol is the most active form of vitamin $\mathrm{E}$ and the most powerful antioxidant (US Department of Agriculture, 2004). There has been some research involving vitamin $\mathrm{E}$ as a possible treatment for PD, but its effects are questionable (Fariss \& Zhang, 2003).

Vitamin $\mathrm{E}$ intake was assessed in three ways: intake of foods containing vitamin $\mathrm{E}$, dietary intake, and total intake including supplements. The risks are reported for quartiles (Hellenbrand et al. 1996a; Anderson et al. 1999; Johnson et al. 1999; Powers et al. 2003), above v. below the median value (Scheider et al. 1997), quintiles (Zhang et al. 2002), and for a continuous variable in mg (Morens et al. 1996).

The CC study of consumption of foods with vitamin E reported a non-significant increased risk of PD (Anderson et al. 1999). There was a significant inverse association in the cross-sectional study between PD and vitamin E intake per $10 \mathrm{mg} / \mathrm{d}$ (de Rijk et al. 1997a). There were no significant risks reported by five $\mathrm{CC}$ studies of total vitamin $\mathrm{E}$. There were no clear trends with publication date or sample size. The Honolulu Heart Study reported vitamin E intake above and below the RDA, OR 0.64 (95\% CI $0.35,1.17)$, and above and below the median, OR 0.90 (95\% CI $0.54,1.48)$ (Morens et al. 1996). 
The NHS and HPFS cohort studies examined vitamin E intake (Zhang et al. 2002). For total vitamin E intake, there was a slightly increased risk for men and decreased risk for women, but both were not significant. There was a significantly decreased risk of PD in women consuming the highest amounts of dietary vitamin E compared with the lowest quintile (Zhang et al. 2002). The decreased risk for men was not significant.

A recent meta-analysis (Etminan et al. 2005) reported a significantly decreased pooled risk for PD with moderate vitamin E intake. Five CC (Morens et al. 1996; Hellenbrand et al. 1996a; Scheider et al. 1997; Anderson et al. 1999; Johnson et al. 1999), one cohort (Zhang et al. 2002) and one cross-sectional study (de Rijk et al. 1997a) reported data for moderate intake but only four of these studies were pooled to assess high intake. The pooled estimates were $0.81(95 \%$ CI $0.67,0.98$ ) for moderate intake (second and third quartile or third and fourth quintile) and 0.78 (95\% CI 0.57, 1.06) with high intake (fourth quartile or fifth quintile).

Total intake of vitamin $\mathrm{E}$ and foods with vitamin $\mathrm{E}$ were not significantly associated with PD in CC studies. The cohort studies indicated a slightly increased risk of PD in men and a lower risk in women with higher vitamin $\mathrm{E}$ intake. This was significant only for dietary vitamin $\mathrm{E}$ intake in women.

\section{Carbohydrates}

Carbohydrates contain sugars, starch, cellulose, gums and related substances (Ensminger 1994). The specific biological hypothesis for an association between carbohydrates and $\mathrm{PD}$ is unclear.

Carbohydrate consumption was reported as: total carbohydrates, disaccharides, monosaccharides, and lactose, and by measuring intake of specific foods that are high in carbohydrates, such as breads, potatoes, cereals and 'other starch foods'. The risk estimates were reported as quartiles (Hellenbrand et al. 1996a,b; Logroscino et al. 1996; Anderson et al. 1999; Johnson et al. 1999; Chen et al. 2002) and quintiles (Chen et al. 2003).

Of three CC studies that measured total carbohydrate consumption, one found a significantly increased OR for PD (Hellenbrand et al. 1996a). The remaining two CC studies failed to find significant associations and reported estimates in opposite directions. All studies adjusted for age, sex, education, and energy intake.

The NHS and HPFS cohort studies reported an increased risk of PD for women and a decreased risk for men with higher total carbohydrate consumption (Chen et al. 2003). Neither estimate was statistically significant.

A German CC study found increased adjusted OR for the highest quartiles of disaccharide and monosaccharide intake, although only the estimate for monosaccharides was significant (Hellenbrand et al. 1996a).

The HPFS male cohort reported a significantly increased $\mathrm{RR}$ for lactose intake of $25 \mathrm{mg}$ or more from diary products, adjusted for $\mathrm{Ca}$, vitamin $\mathrm{D}$, fat and protein (Chen et al. 2002).

There were no significant risks reported for cereals or 'other starch foods'. A non-significant decreased OR was reported by one $\mathrm{CC}$ study for combined bread and cereal intake (Anderson et al. 1999). Two sex-specific cohort studies measured cereal intake and other starch foods, but did not find significant associations (Chen et al. 2002). One CC study reported a significantly decreased unadjusted OR for individuals consuming French loaf or wheat bread, daily v. never (Fall et al. 1999). Another CC study found a significantly decreased risk for PD among individuals consuming the highest quartile of potatoes (Hellenbrand et al. 1996b). All studies were adjusted for age, sex, and other covariates.

Higher intake of total carbohydrates and sugars was associated with PD in some CC studies. The finding was not confirmed in cohort studies, which found no significant effects, with the exception of a positive association with lactose intake in men. Specific foods high in carbohydrates were not associated with PD except for potatoes and French or wheat bread, which were inversely associated with PD.

\section{Fats and cholesterol}

Fats and oils are lipids, which are involved with fat-soluble vitamin transport among other functions. The intake of excess fats may be relevant to PD aetiology because unsaturated fatty acids are the targets of lipid peroxidation, which can generate free radicals and cause oxidative stress (Ensminger, 1994). Other functions of the fatty acids, such as the role of arachidonic acid in inflammatory response, may also be involved in PD. The biological relationship of cholesterol to PD aetiology is not clear.

Fish oils are a rich source of n-3 long-chain PUFA, including EPA and DHA. DHA may play a role in neurological function and/or neuroprotection, although its specific function is undefined (Salem et al. 2001). Arachidonic acid may have anti-inflammatory effects, as shown in studies of rheumatoid arthritis patients (Cleland et al. 2003).

Fat intake was measured as total, animal, dairy, vegetable, saturated, monounsaturated, polyunsaturated, and transunsaturated. Specific fatty acids were examined in the NHS and HPFS cohorts: arachidonic, docosahexaenoic, eicosapentaenoic, linoleic, linolenic, oleic, and n-3 (Chen et al. 2003). The risk estimates for fats were reported as quartiles (Logroscino et al. 1996; Hellenbrand et al. 1996a; Anderson et al. 1999; Johnson et al. 1999; Powers et al. 2003), quintiles (Chen et al. 2003), and one or more servings $v$. none (Morens et al. 1996). Total intake of cholesterol was measured by three studies (Johnson et al. 1999; Chen et al. 2003).

Of four CC studies examining total fat intake, only one found a significant positive association with PD (Hellenbrand et al. 1996a; Logroscino et al. 1996; Johnson et al. 1999; Powers et al. 2003). All studies adjusted for age, sex, and energy intake. There was no trend in the estimates with publication date; however, the two studies with smaller sample sizes reported positive associations with fat consumption, whereas the two larger studies reported inverse associations. The NHS and HPFS cohorts reported an increased risk of PD with higher fat consumption for men, and a reduced risk for women. Neither result was significant. 
In addition to total fat, studies examined the relationship between specific subtypes of fat and PD. Only the HPFS cohort reported fat intake categorised by dairy and nondairy sources. Both RR were non-significant, adjusted for Ca, vitamin D, protein and lactose (Chen et al. 2002). Two $\mathrm{CC}$ studies measured intake of animal fat and both found significant positive associations, adjusted for age, sex, energy intake, and other covariates. One male NCC study reported a non-significant estimate in the same direction, adjusted for smoking and coffee (Morens et al. 1996). The NHS and HPFS cohorts found an increased RR for PD in men and a decreased risk in women comparing highest and lowest quintiles of animal fat intake, although neither result was significant (Chen et al. 2003).

The only CC study to report an OR for fats from vegetables found no difference in intake between cases and controls (Logroscino et al. 1996). A male NCC study found a nonsignificant reduced risk with at least one serving of vegetable oil compared with none (Morens et al. 1996). The HPFS cohort found a non-significant reduced risk of PD for men consuming vegetable fat in the highest quintile (Chen et al. 2003). The NHS cohort found a non-significant increased RR for women in the highest quintile (Chen et al. 2003).

Two CC studies reported OR in opposite directions for saturated fat intake. The larger of the two found a significantly reduced OR, whereas the study with approximately half the number of cases found a non-significant increased OR (Johnson et al. 1999; Powers et al. 2003). Both studies, conducted in the USA, were adjusted for age, sex, race, smoking and energy intake, but other covariates differed. The HPFS and NHS cohort studies reported an increased risk of PD for men and a decreased risk for women, and both were non-significant (Chen et al. 2003).

Only the HPFS and NHS cohorts examined the relationships of monounsaturated, polyunsaturated and trans-unsaturated fat with PD (Chen et al. 2003). None of the RR were significant. For monounsaturated fat, men appeared to have a slightly increased risk and women a decreased risk for the highest intake.

The NHS and HPFS cohorts reported inverse relationships between arachidonic acid intake and PD in men and women, although only the pooled estimate reached statistical significance (Chen et al. 2003). The RR for DHA, EPA, and n-3 fatty acids were below unity but nonsignificant.

The NHS and HPFS cohorts reported a non-significant inverse relationship between $\mathrm{PD}$ and linoleic acid intake (Chen et al. 2003). One CC study also measured linoleic acid intake and reported no association with PD (Johnson et al. 1999). The same CC study reported a non-significant increased OR for oleic acid intake. The RR from the NHS and HPFS for linolenic acid intake was above unity for women and below for men, although neither was statistically significant (Chen et al. 2003).

The CC study that estimated cholesterol intake found a non-significant positive association with PD (Johnson et al. 1999). The HPFS and NHS studies reported non-significant RR in opposite directions (Chen et al. 2003). The Honolulu Heart Study measured serum cholesterol (mg) in men at baseline and found no significant difference in the RR of PD. This measure may not be directly related to cholesterol consumption as the body also produces cholesterol (Grandinetti et al. 1994).

Two cohort studies and one NCC study did not find significant associations between $\mathrm{PD}$ and fat or specific types of fat. Two CC studies found significantly increased OR for the highest levels of total and/or animal fat intake. The risk estimates for specific fatty acids were not significant. There was no evidence to suggest that cholesterol intake is related to PD.

\section{Proteins}

Proteins are complex organic molecules made up of amino acids. Some functions of proteins are cell maintenance, regulation, and energy formation. The specific biological hypothesis for an association between proteins and PD is unclear.

Protein intake was measured as total protein and by foods that are mainly composed of protein. The risk estimates for proteins were reported as quartiles (Hellenbrand et al. 1996a; Logroscino et al. 1996; Anderson et al. 1999; Johnson et al. 1999; Chen et al. 2002), quintiles (Chen et al. 2003), and by frequency of consumption (Fall et al. 1999).

Total protein intake was measured by three CC studies, and none found a significant OR (Hellenbrand et al. 1996a; Logroscino et al. 1996; Johnson et al. 1999). The two larger studies reported an inverse association with PD, whereas the smaller study reported a two-fold increased risk. The smaller study matched controls by frequency, whereas the other two matched each individual case. All adjusted for age and sex. The HPFS and NHS cohorts reported nonsignificant RR for protein intake (Chen et al. 2003).

A CC study reported an inverse association between PD and total meat intake (Anderson et al. 1999). The same study found a positive association for red meat consumption. The HPFS cohort reported non-significant increased $\mathrm{RR}$ in men for total meat and red meat (Chen et al. 2003). In contrast, the NHS cohort reported non-significant decreased risks in women (Chen et al. 2003).

One unmatched, unadjusted CC study reported nonsignificant decreased OR for both fried and broiled meat, and smoked ham and meat, comparing daily consumption with never (Fall et al. 1999). The study found a significantly decreased risk of PD for individuals consuming eggs daily compared with never.

The HPFS and NHS cohorts found no significant association between PD and chicken consumption, although estimates indicated a protective effect with higher intake (Chen et al. 2003). The RR for fish consumption was similar to chicken in men, but in women the RR was nonsignificantly increased for the highest quartile of consumption. A CC study measuring combined chicken and fish consumption reported a non-significant decreased OR (Anderson et al. 1999). The same study found no association for fish intake alone. The cohort studies and CC study were adjusted for age, sex, energy intake and other covariates.

In general there were no significant associations or patterns for the relationship between protein intake and PD. The cohort RR were close to unity, and the CC study OR were in both directions. The most extreme estimates, nearly reaching significance, were for inverse associations between 
PD and fried and broiled meat consumption, and PD and smoked ham and meat consumption. However, the estimates were from one unadjusted CC study; therefore they must be interpreted with caution.

\section{Dairy products}

Dairy is comprised of all products made from milk. Milk contains carbohydrates, proteins, minerals, fat and vitamins. There are no specific a priori hypotheses for dairy products.

One CC and three cohort studies examined the association of PD with total dairy product or milk intake (Anderson et al. 1999; Chen et al. 2002; Park et al. 2005). More specific dairy product analyses were conducted in the HPFS male cohort only (Chen et al. 2002). There were no significant risk estimates for the highest compared with the lowest quartiles of total dairy consumption in the CC study or the female NHS cohort, although the estimates were in opposite directions. The risk was significantly elevated in men consuming the highest amount of total dairy products in both the HPFS and Honolulu male cohorts. Ca had no apparent association with PD after adjustment for milk consumption (Park et al. 2005). For the sub-categories high-fat and low-fat dairy, both the HPFS and NHS cohorts had increased but non-significant RR with higher consumption. The PD risk estimates for specific dairy products in HPFS men were not significant, with the exception of an elevated risk in men consuming cream cheese at least once per week compared with never.

Dairy product intake was examined by only four studies, and a significant association between total dairy intake and PD was found in the two male cohorts only. A sub-analysis of specific dairy products in the HPFS cohort suggested that cream cheese and sour cream intake more than once per week compared with never might increase risk of PD.

\section{Alcohol intake}

Alcohol is usually consumed in the form of ethanol. Alcohol is included in the present review as a beverage type. Alcohol is combined with $\mathrm{O}_{2}$ in the liver, where alcohol is changed into the toxic chemical acetaldehyde. This is converted to acetate in the liver and other cells. Acetate is oxidised to $\mathrm{CO}_{2}$ and water to produce heat and energy (Ensminger, 1994).

The brain is very sensitive to alcohol. At low levels in the blood it may act as a stimulant, but its main effect is as a depressant. Alcoholism can cause premature ageing of the brain, which may cause temporary or permanent damage to cognitive function (Ensminger, 1994). It is also possible that alcohol contributes to oxidative stress, which may affect the brain (Collins, 2002). However, alcohol has been discussed as a protective factor for neurodegeneration and vascular disease, although the mechanisms are unknown (Desagher et al. 1996; Gonthier et al. 2004).

The intake of alcohol was measured as: 'ever $v$. never'; 'yes v. no'; ex- or current drinker $v$. never drinkers; heavy or moderate intake $v$. never drinkers; quantitative comparisons using estimated alcohol or ethanol consumed $(\mathrm{g})$ per $\mathrm{d}$; and durations of exposure.

The binary measures were combined and summarised using a meta-analysis comparing drinkers with nondrinkers. Information was available from thirteen CC studies (Haack et al. 1981; Ho et al. 1989; JimenezJimenez et al. 1992; Mayeux et al. 1994; Liou et al. 1997; Smargiassi et al. 1998; Gorell et al. 1999; Nelson et al. 1999; Benedetti et al. 2000; Behari et al. 2001; Checkoway et al. 2002; Tsai et al. 2002; Ragonese et al. 2003) and four cohort studies (Grandinetti et al. 1994; WillemsGiesbergen et al. 2000; Hernan et al. 2003).

The meta-analysis results are reported for the fixed effects models, as the random and fixed effects estimates were similar. The CC pooled OR was 0.81 (95\% CI 0.70, 0.92) (Fig. 1). The estimates for Tsai and Nelson were for current drinkers v. never, as overall estimates could not be calculated (Nelson et al. 1999; Tsai et al. 2002). The pooled estimate excluding these two studies was $0.86(95 \%$ CI $0.74,1.00)$. The cohort pooled RR estimate was 0.73 (95\% CI 0.57, 0.92) (Fig. 2). Two of the four cohorts were

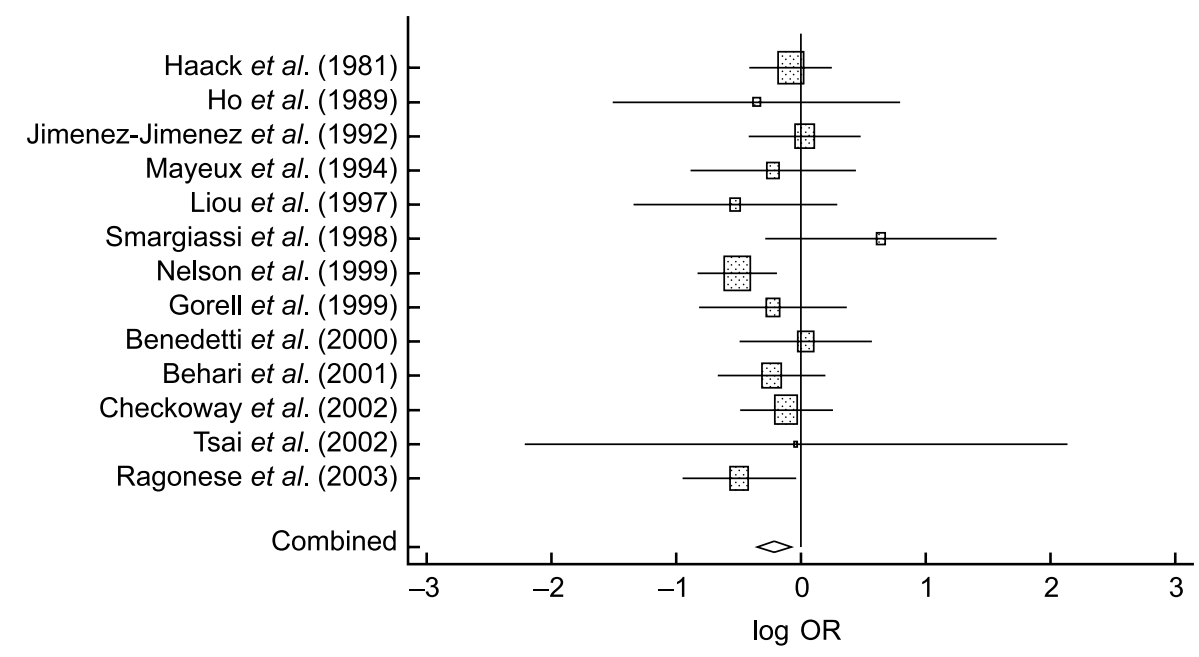

Fig. 1. A meta-analysis of the association between ever drinking alcohol and Parkinson's disease in thirteen case-control studies. For Nelson et al. (1999) and Tsai et al. (2002), the estimates represent current v. never drinking alcohol, as the overall estimate could not be calculated. 


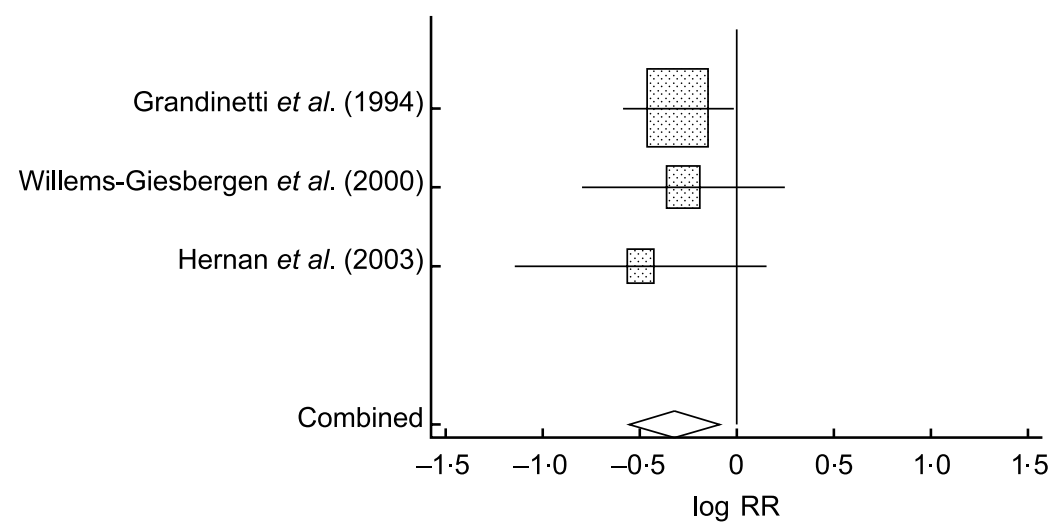

Fig. 2. A meta-analysis of the association between ever drinking alcohol and Parkinson's disease in three cohort studies. Hernan et al. (2003) is a pooled estimate of the Nurses' Health Study and Health Professionals' Follow-up Study sex-specific cohorts.

sex specific but a pooled estimate was available and used for the meta-analysis (Hernan et al. 2003).

In general the OR estimates indicated an inverse association, although only two were significant (Nelson et al. 1999; Ragonese et al. 2003). Three non-significant OR indicated a positive relationship between alcohol drinking and PD, two of which were in women only (JimenezJimenez et al. 1992; Smargiassi et al. 1998; Benedetti et al. 2000). The four cohort studies reported inverse associations, but only the unadjusted estimate from the male HPFS cohort reached borderline significance.

The five $\mathrm{CC}$ studies using semi-quantitative measures, which estimated ethanol intake $(\mathrm{g})$ or number of drinks, reported a general inverse relationship with PD (JimenezJimenez et al. 1992; Wang et al. 1993; Hellenbrand et al. 1996b; Checkoway et al. 2002; Ragonese et al. 2003). The estimates from two of the studies reached statistical significance. The NCC study and two cohorts reported the same inverse relationship but only the NCC study was significant (Paganini-Hill, 2001; Hernan et al. 2003).

One CC study reported OR for alcohol drinking stratified by the number of years drinking compared with nondrinkers. There was a non-significant inverse association between PD and drinking for less than or equal to 20 years. The relationship was reversed for those consuming alcohol for more than 20 years (Behari et al. 2001).

Alcohol intake was categorised in several studies by specific types of alcoholic beverages: beer, wine and spirits or liquor.

Two CC studies reported significant inverse associations between PD and beer intake. One measured consumption in quartiles (Hellenbrand et al. 1996b) and the other stratified beer intake by strength, medium and strong, and compared the number of bottles consumed with the reference category of one or less bottle of beer per d and week, respectively (Fall et al. 1999). The HPFS and NHS cohorts reported a significant inverse association between PD and the consumption of one or more beers per week compared with less than one per month (Hernan et al. 2003). However, the RR for the male and female cohorts separately were not significant.

The measurement of wine consumption differed between studies and the estimates were similarly variable. The larger of two CC studies reported a non-significant increased OR for PD comparing highest and lowest quartiles of wine consumption (Hellenbrand et al. 1996b). The other study, unmatched and unadjusted, reported a significantly decreased risk for drinking two to six bottles of wine per week compared with non-drinkers (Fall et al. 1999). The pooled RR for the HPFS and NHS cohorts was nonsignificantly increased for drinking wine at least five times per week compared with less than once per month. The risk was increased in women and decreased in men, but neither was significant.

Hard liquor, liquor and spirits were considered the same exposure. All three CC studies reported significant inverse associations between PD and liquor consumption (Wang et al. 1993; Hellenbrand et al. 1996b; Fall et al. 1999). The two smaller studies measured liquor as a binary exposure, yes or no, and by the number of bottles consumed per week (Wang et al. 1993; Fall et al. 1999). The largest study compared the highest and lowest tertiles of intake (Hellenbrand et al. 1996b). The pooled RR for the NHS and HPFS cohorts was non-significant comparing individuals consuming liquor five or more times per week with less than once per month (Hernan et al. 2003). The cohorts reported non-significant risks for men and women separately.

Overall there is some evidence for an inverse association between alcohol and PD; however, the majority of studies failed to reach statistical significance. Of thirteen CC studies comparing drinkers with non-drinkers, ten showed an inverse association, two of which were significant. The four cohort studies all showed inverse associations, although only an unadjusted estimate for men was borderline significant. When alcohol was measured quantitatively, all OR for five CC studies were below one, two of which were significant. The NCC showed a significant inverse association, and the two cohorts reported non-significant inverse associations.

\section{Coffee, tea and caffeine}

Caffeine is a drug with primarily stimulatory effects (Ensminger, 1994). Caffeine is mainly metabolised by the CYP1A2 isoenzyme of the p450 family (Fredholm et al. 
1999; Pollock et al. 1999). The effects of caffeine include stimulation of the central nervous system (Weinreb et al. 2004).

A meta-analysis of eight CC studies (Nefzger et al. 1968; Haack et al. 1981; Jimenez-Jimenez et al. 1992; Morano et al. 1994; Fall et al. 1999; Benedetti et al. 2000; Preux et al. 2000; Paganini-Hill, 2001) and four cohort studies (Ross et al. 2000; Ascherio et al. 2001; Fink et al. 2001) that investigated the association between coffee drinking and PD reported a pooled estimate of $0.74(95 \%$ CI $0.67,0.81)$ for the fixed effects model and $0.72(95 \%$ CI $0.64,0.81)$ for the random effects model (Hernan et al. 2002). These estimates were corrected after the original publication (MA Hernan, personal communication). The pooled RR per three additional cups of coffee per d was 0.75 (95\% CI 0.64, 0.86) in CC studies (Nefzger et al. 1968; Jimenez-Jimenez et al. 1992; Benedetti et al. 2000; Ahmadi et al. 2000), and 0.68 (95\% CI 0.46, 1.00) in cohort studies (Ross et al. 2000; Willems-Giesbergen et al. 2000; Ascherio et al. 2001).

Coffee drinking overall was found to be protective for PD. The pooled estimates for CC and cohort studies were not significantly different from each other. None of the individual studies reported increased risks, but some of the protective risk estimates were not statistically significant.

The inclusion and exclusion criteria for the meta-analysis were similar to the criteria set out for the present systematic review. Two studies not included in the meta-analysis were identified from the specified time period (Zayed et al. 1990; Hellenbrand et al. 1996b). Hellenbrand was mentioned but not included because the data did not provide enough information to calculate OR. Zayed reported a nonsignificant inverse relationship for the binary and quantitative measures of coffee intake (Zayed et al. 1990; Hellenbrand et al. 1996b).

Three additional CC studies were published after the meta-analysis inclusion period (Checkoway et al. 2002; Ragonese et al. 2003; Tan et al. 2003). Tan would not have been included because the exposure, thirty cup-years, cannot be calculated in binary terms. The other two studies reported inverse associations between PD and coffee drinking, but only Ragonese was significant. The additional studies would not significantly change the pooled estimates from the 2002 meta-analysis.

The quantitative measurements compare the highest and lowest categories of coffee consumption in seven $\mathrm{CC}$, five cohort and one NCC studies. All but two specified the number of cups per d (Hellenbrand et al. 1996b; Ross et al. 2000). Hellenbrand compared quartiles and Ross computed an RR using ounces of coffee consumed. Zayed used a combined measure of coffee and tea consumption (Zayed et al. 1990). All risk estimates supported the inverse relationship between coffee and PD. Three CC (Hellenbrand et al. 1996b; Fall et al. 1999; Benedetti et al. 2000), one cohort (Ross et al. 2000) and the NCC (Paganini-Hill, 2001) study reported significant inverse associations.

Two age- and sex-matched CC studies measured coffee cup-years and reported OR significantly below unity (Ragonese et al. 2003; Tan et al. 2003). The OR for individuals consuming greater than thirty cup-years, estimating three cups per d for 10 years, was $0.79(95 \%$ CI 0.66, 0.93) (Tan et al. 2003). The analysis was adjusted for covariates including smoking, alcohol and tea. The other study reported OR of 0.36 (95\% CI $0.05,1.86), 0.19$ (95\% CI $0.08,0.43)$ and $0.20(95 \%$ CI $0.08,0.47)$ for one to thirtyeight, thirty-nine to eighty and eighty-one to 288 cup-years, respectively (Ragonese et al. 2003). The estimates were adjusted for education but not for smoking. The results support the inverse association between coffee drinking and PD.

Two studies reported non-significant OR for the relationship between decaffeinated coffee and PD. The CC study did not find an association between PD and regular or decaffeinated coffee. The decaffeinated OR was $1.10(95 \%$ CI $0 \cdot 7,1.8)$ (Checkoway et al. 2002). The NCC study found a significant inverse relationship between $\mathrm{PD}$ and the consumption of two or more cups of regular coffee compared with non-drinkers (Paganini-Hill, 2001). This same comparison with decaffeinated coffee produced a null result, RR $0.94(95 \%$ CI $0.72,1.23)$. If this result is replicated it might indicate that caffeine is the exposure affecting the outcome of PD.

Tea drinking exposure was measured as yes or no (Ho et al. 1989; Yang et al. 1994; Morano et al. 1994; Preux et al. 2000), cups per d (Chan et al. 1998; Fall et al. 1999; Paganini-Hill, 2001; Checkoway et al. 2002) and cup-years (Tan et al. 2003). The risk estimates for PD and tea consumption are inconsistent. The only NCC study reported a non-significant increased OR comparing drinking at least two cups of tea per d to none (Paganini-Hill, 2001).

Four CC studies measured tea consumption as a binary variable, and there were two OR estimates in each direction (Ho et al. 1989; Yang et al. 1994; Morano et al. 1994; Preux et al. 2000). The only estimate reaching significance reported a positive association between tea drinking and PD (Preux et al. 2000).

All four CC studies with quantitative measures of tea drinking reported significant inverse associations with PD (Chan et al. 1998; Fall et al. 1999; Checkoway et al. 2002; Tan et al. 2003). The NCC found a non-significant positive association.

There is no clear trend of the risk estimates with publication date or geographical location. Of the three studies that reported increased risk of PD with tea drinking, one was unadjusted and two were adjusted for age and sex. Of the six studies reporting a decreased risk, three were unadjusted and three were adjusted for age, sex and other covariates.

One CC study investigated the association between cola intake and PD, and the risk estimate was 0.60 (95\% CI 0.30, $1 \cdot 40$ ), adjusted for age, sex and clinic (Checkoway et al. 2002).

The Honolulu Heart Study male cohort used dietary food and beverage consumption to estimate total caffeine intake and caffeine from non-coffee sources. The RR for caffeine in the highest $v$. lowest quintile was 0.20 (95\% CI 0.08 , $0 \cdot 48)$. For caffeine from non-coffee sources the RR was $0 \cdot 37$ (95\% CI $0 \cdot 19,0 \cdot 71)$. Both estimates were adjusted for age and smoking (Ross et al. 2000).

All ten CC, one NCC and four cohort studies reported inverse relationships between PD and ever drinking coffee. Four of the $\mathrm{CC}$ estimates were significant, as were those from the NCC and the male HPFS cohort. All OR and RR 
using quantitative measures were less than or equal to unity, with the one $\mathrm{NCC}$, four of seven $\mathrm{CC}$, and one of five cohort studies reporting significant associations.

There were two OR estimates in each direction for the binary measure of tea drinking, and only one suggesting a positive association with PD was significant. All CC studies measuring tea consumption quantitatively reported significant inverse associations.

Overall the evidence points to an inverse association between PD and caffeine or caffeinated beverages, although not entirely.

\section{Other foods}

Three studies examined the association between junk foods and PD. One CC study found a non-significant decreased OR for higher junk food intake (Anderson et al. 1999). A larger CC study reported significantly increased OR for higher consumption of sweets, chocolate, and desserts (Hellenbrand et al. 1996b). The HPFS and NHS cohorts did not find significant risks for increased consumption of 'chocolate, candies or brownies' or 'other sweets or desserts' (Chen et al. 2002).

The OR for the associations between PD and other miscellaneous foods were mostly non-significant. The intake of nuts and seeds was positively associated with PD in a CC study, but the HPFS and NHS cohorts combined reported a significantly decreased risk for higher nut intake (Butterfield et al. 1993; Zhang et al. 2002).

\section{Calcium}

$\mathrm{Ca}$ is an essential element whose absorption depends on dietary factors such as vitamin $\mathrm{D}$, lactose, protein and acid medium. Ca plays a role in nerve impulse transmission (Ensminger 1994). No a priori hypotheses appear to be discussed for $\mathrm{Ca}$.

$\mathrm{Ca}$ intake was measured as total dietary $\mathrm{Ca}$ and $\mathrm{Ca}$ supplements. Two CC studies reported non-significant increased OR comparing the highest with lowest quartiles of dietary Ca intake (Johnson et al. 1999; Powers et al. 2003). The HPFS male cohort reported a non-significant protective effect of Ca supplement intake above $200 \mathrm{mg} / \mathrm{d}$ compared with none (Chen et al. 2002).

Overall, there were no significant associations between $\mathrm{Ca}$ intake and PD.

\section{Iron}

Fe is an essential mineral and is a component of proteins involved in $\mathrm{O}_{2}$ transport and metabolism (Institute of Medicine, 2001). The role of $\mathrm{Fe}$ in PD has been investigated because its levels are increased in the PD substantia nigra, and it has the capacity to enhance the production of oxygen free radicals (Berg et al. 2001).

$\mathrm{Fe}$ intake was measured as: foods with $\mathrm{Fe}$, dietary $\mathrm{Fe}$ and total Fe including supplements. Of four CC studies, two reported borderline positive associations with $\mathrm{PD}$. The smallest study reported a non-significant inverse association between PD and the consumption of foods with Fe (Anderson et al. 1999). Another small study reported a nearly significant positive association between PD and dietary $\mathrm{Fe}$ intake. However, when dietary $\mathrm{Fe}$ and supplements were considered together, the OR was nonsignificant and less than unity. Two larger CC studies considering the total intake of $\mathrm{Fe}$ reported positive associations, one of which was nearly significant.

The two smallest studies reported estimates in opposite directions for dietary intake without supplements. The first study was unmatched but adjusted for age, sex and other covariates (Anderson et al. 1999). The second study was frequency matched for age, ethnicity and sex, but unadjusted in the analysis (Logroscino et al. 1998).

For total Fe intake including supplements, two studies reported increased OR (Johnson et al. 1999; Powers et al. 2003) and one reported a decreased OR (Logroscino et al. 1998). The two positive studies were larger, frequencymatched for age and sex, and adjusted for various covariates.

There is a possible positive association between $\mathrm{Fe}$ and PD. Two CC studies reported borderline significant positive associations between $\mathrm{Fe}$ intake and PD, although one found a null result for dietary and supplemental Fe combined. The two remaining studies reported non-significant estimates in opposite directions.

\section{Multivitamins or total vitamins}

Multivitamin intake is a heterogeneous exposure and intake was measured as: vitamins, vitamin or cod liver oil supplement, and multivitamins. One CC study reported a non-significant increased $\mathrm{OR}$ for $\mathrm{PD}$ with the intake of vitamin or cod liver oil supplements at least once per week (Chan et al. 1998). Another CC study reported no association with vitamin intake (Taylor et al. 1999). The HPFS and NHS cohort studies analysed multivitamin intake for men and women, separately and pooled (Zhang et al. 2002). None of the comparisons were significant.

Overall, there were no significant associations between vitamin or multivitamin use and PD. The results from the analyses of past or current use in the NHS and HPFS cohorts indicate that past use but not current use for greater than 10 years may be protective for PD in men, and the opposite relationship is indicated for women.

\section{$B$ vitamins}

$\mathrm{B}$ vitamins are often referred to by their most common forms: vitamin $\mathrm{B}_{1}$ (thiamin); vitamin $\mathrm{B}_{2}$ (riboflavin); vitamin $\mathrm{B}_{3}$ (niacin); vitamin $\mathrm{B}_{5}$ (pantothenic acid); vitamin $\mathrm{B}_{6}$ (pyridoxine); and vitamin $\mathrm{B}_{12}$ (cobalamin).

$\mathrm{B}_{6}$ is a water-soluble vitamin whose major function is in amino acid metabolism, and extremely high intakes have been associated with neurological damage (Bender, 1989).

Folate and folic acid are different forms of a water-soluble B vitamin (Herbert, 1999). Levels of folate and homocysteine are inversely related (Verhoef et al. 1996). Low folate status may play a role in depression, cognitive impairment and/or dementia, although evidence is inconsistent and the causal relationship has not been proven (Morris, 2002). A study in mice found that folate deficiency caused increased susceptibility to 1-methyl-4-phenyl-1,2,3,6-tetrahydropyridine, 
a chemical known to cause Parkinsonian signs (Miller, 2002). Folate also plays a role in the formation of purines and pyrimidines necessary for RNA and DNA synthesis. Vitamin $\mathrm{B}_{12}$, or cobalamin, is necessary for DNA synthesis (Herbert, 1996).

Two CC and two cohort studies examined the intake of various B vitamins (Hellenbrand et al. 1996a; Johnson et al. 1999; Chen et al. 2004). The NHS and HPFS cohorts measured folate, vitamins $\mathrm{B}_{6}$ and $\mathrm{B}_{12}$ as total, dietary and supplement intake. Total and dietary intakes were divided into quintiles. Supplemental intake was divided into three categories and compared non-users with users above and below specified amounts of intake (Chen et al. 2004).

The relationship between thiamin intake and PD was examined by one CC study (Johnson et al. 1999). The adjusted OR was non-significant, comparing highest and lowest quartiles.

The CC studies reported estimates in opposite directions comparing the highest and lowest quartiles of riboflavin, folate and pyridoxine intake, separately. The US study estimates were above one and non-significant, whereas the German study OR were significantly less than one for all three vitamins (Hellenbrand et al. 1996a; Johnson et al. 1999). The two cohort studies reported non-significant RR for total, dietary and supplemental folate and vitamin $\mathrm{B}_{6}$ intake (Chen et al. 2004).

Both CC studies reported decreased OR for niacin, although only the larger study was significant. Hellenbrand found a significantly decreased OR for pantothenic acid and a non-significant decreased OR for cobalamin. The two cohort studies reported non-significant RR for total, dietary and supplemental cobalamin intake (Chen et al. 2004).

The CC studies were matched for age and sex, and adjusted for multiple covariates including energy intake. The German study (Hellenbrand et al. 1996a) was twice as large as the American study (Johnson et al. 1999). Both studies measured nutrient intake with a food-frequency questionnaire and specified PD diagnostic criteria. One difference in case selection was that Hellenbrand chose individuals under 67 years, and Johnson chose individuals greater than 50 years of age. The sex-specific cohort studies adjusted for age, sex, and total energy and other factors.

In summary, two CC and two cohort studies examined the association between B vitamins and PD. The cohort studies reported no significant associations comparing the highest and lowest quintiles of folate, vitamin $\mathrm{B}_{6}$ or vitamin $\mathrm{B}_{12}$ intake. In the $\mathrm{CC}$ studies, there were no significant associations between PD and thiamin, riboflavin, and cobalamin. The German study reported an inverse relationship between niacin and $\mathrm{PD}$, which was supported by the non-significant finding in the US study. The CC studies had estimates in opposite directions for pyridoxine and folate. The German study reported significant inverse associations, and the US study reported non-significant positive associations. The German study found a significant inverse relationship between pantothenic acid and PD.

\section{Total energy}

It has been observed that PD patients consume more energy than unaffected individuals while still losing body weight
(Davies et al. 1994). This may be due to their using more energy for activities of daily living; however, it is possible that this difference could also have existed in the premorbid diet. No hypotheses have been discussed for the role of energy intake in PD. Six studies compared the energy intake between PD cases and unaffected individuals (Hellenbrand et al. 1996b; Logroscino et al. 1996; Anderson et al. 1999; Johnson et al. 1999; Chen et al. 2003).

The four CC studies compared the highest and lowest quartiles of energy intake. Three reported increased OR, of which two were significant. The fourth study reported a nonsignificant estimate below unity. All were adjusted for age and sex, as well as other factors.

The NHS and HPFS cohort studies reported a pooled adjusted RR of one comparing the highest and lowest quintiles of energy intake. The NHS was slightly below, and the HPFS slightly above null, but neither was significant.

Overall, the CC studies suggested a positive association between energy intake and PD, but this was not supported by the null findings in the two cohort studies.

\section{Miscellaneous nutrients}

Only nutrients that were reported by at least three unrelated studies were summarised in separate sections and the rest are reported below.

$\mathrm{K}$ maintains cellular osmotic pressure and acid-base balance. $\mathrm{Na}$ is associated with muscle contraction and nerve function, and it plays a role in carbohydrate absorption. $\mathrm{P}$ is involved in almost all metabolic functions and is a component of nucleic acids (Ensminger 1994).

One CC study reported a non-significant reduced OR for $\mathrm{K}$, and a non-significant increased $\mathrm{OR}$ for $\mathrm{Na}$ and $\mathrm{P}$ (Johnson et al. 1999). All estimates were adjusted for age, sex, race, total energy and smoking.

$\mathrm{Cu}$ is a constituent of enzyme systems that relate to brain cell and spinal cord function. $\mathrm{Cu}$ deficiency can lead to disorders including dymelination and degeneration of the nervous system. $\mathrm{Mg}$ is an essential mineral required for the activation of enzymes involved with the phosphorylation of ADP to ATP (Ensminger 1994). Mg releases nerve impulses and is involved with muscle contraction (US Department of Agriculture, 2004). Zn is an essential mineral necessary for synthesis and metabolism of proteins and nucleic acids. Se is an essential trace mineral; it is an important part of antioxidant enzymes (Combs \& Gray, 1998). Mn is involved in cholesterol synthesis and the metabolism of carbohydrates, fats, proteins and nucleic acids (Ensminger, 1994).

One CC study reported no significant associations between $\mathrm{PD}$ and $\mathrm{Cu}, \mathrm{Mg}, \mathrm{Mn}$, Se or $\mathrm{Zn}$ intake, comparing the highest and lowest quartiles of intake (Powers et al. 2003). One female cohort study examined Mn intake and reported a significant inverse association comparing highest and lowest tertiles (Cerhan et al. 1994). This estimate was in the opposite direction of the other study. Both studies were adjusted for age and education, and the CC study was further adjusted.

It has been hypothesised that uric acid might have a role in protecting against free radical oxidation (Ames et al. 1981; Tohgi et al. 1993; Church \& Ward, 1994; Davis et al. 1996). The Honolulu Heart Study male cohort reported 
a non-significant reduced risk for PD for higher levels of serum uric acid, adjusted for age and smoking (Davis et al. 1996). The biological measure was objective and is assumed to reflect intake.

Vitamin D, or calciferol, is a fat-soluble vitamin whose primary function is to maintain $\mathrm{Ca}$ and $\mathrm{P}$ levels in the blood (Institute of Medicine, 1999).

A CC study reported a non-significant increased OR for the highest $v$. lowest quartile of intake for foods with vitamin D, with and without supplements (Anderson et al. 1999). The HPFS male cohort study reported a nonsignificant result in the same direction for supplement intake of greater than $10 \mu \mathrm{g}$ (400 IU) compared with none. The RR was the same for 0.025 to $9.975 \mu \mathrm{g}$ (1 to $399 \mathrm{IU}$ ), indicating a lack of dose response (Chen et al. 2002).

\section{Discussion}

\section{Systematic review methods}

The searches for previous systematic reviews on the topic of nutritional risk factors for PD were limited to the years 1989 until 2004. Before 1989, systematic reviews were not widely conducted, and older reviews would need to be updated with literature from more than 15 years. One meta-analysis including articles published between 1966 and 2002 reported an inverse association between coffee drinking and PD (Hernan et al. 2002). A systematic review of $\beta$-carotene, vitamin $\mathrm{E}$, and vitamin $\mathrm{C}$ from literature published between 1996 and 2005 reported no associations between PD and vitamin $\mathrm{C}$ or $\beta$-carotene, but a significant inverse association between vitamin $\mathrm{E}$ intake and PD (Etminan et al. 2005).

The MEDLINE, EMBASE and WEB OF SCIENCE databases were used to search for original contributions to the topic. Additional articles were gathered by scanning the bibliographies of the primary articles. No attempt was made to obtain unpublished results. The quality of unpublished results cannot be easily assessed, and the value of obtaining such data was believed to be limited, although publication bias does make it probable that a substantial proportion of negative studies would exist.

Inclusion and exclusion criteria for the review were clearly specified to ensure the quality of included articles. Cross-sectional studies were to be excluded but the results are reported from one study because the methods were similar to CC studies (de Rijk et al. 1997a).

Data extraction was conducted using a standardised form by one author (L. I.). Qualitative details were recorded using recommended methods. The quality scores were not used for qualitative analysis but to determine the study characteristics that could be the sources of heterogeneity (Tables $1-3$ ).

Most data are presented as a systematic review and summary of results rather than a meta-analysis. This was due to the heterogeneity in exposure measurement and other study design characteristics, and the limited numbers of studies examining specific exposures.

\section{Study methods}

Subject selection. The selection of cohorts is important because the study population can determine both the validity and generalisability of the findings. A specific group of individuals may have better response and follow-up rates at the cost of generalisability to the total population of interest, and vice versa.

Case and control selection influence the validity and generalisability of results (Table 2). The PD patients were recruited through sources, most of which relied on the presence of a PD diagnosis. The movement disorders clinics and neurology practices may treat a higher proportion of severe PD patients than those identified from records. Secondary and drug-induced Parkinsonism were for the most part excluded; however, this is dependent on the clinical history, and cases that have been classified as PD may affect the risk estimates.

Controls were matched in most of the studies. PD is a disease related to ageing; therefore matching by age is important. Preliminary findings in the literature suggest a male preponderance; therefore sex might also be a key matching criterion. Matching by other variables may reduce additional confounding.

The selection and response rate of controls must be considered for generalisability to the overall population. The most unbiased method of selection is random selection from the community. Hospital controls and elderly home residents may be more ill than the general population. Friends or spouses may be too similar in environmental exposures (for example, smoking, alcohol, caffeine) to examine differences.

Some studies excluded individuals with cognitive impairment and/or dementia. This criterion may exclude older and more severe PD cases, since PD is a risk factor for dementia (Aarsland et al. 2003). Other studies restricted the age group for cases. This could be useful if the early- and late-onset cases differ in aetiology. There is some evidence that this is the case because genes implicated in early-onset familial PD are not found in later-onset sporadic PD (Gwinn-Hardy, 2002). Another way to examine the effect of age would be to stratify analyses rather than exclude individuals.

Study size and power. There were very few significant results reported amongst the many nutritional factors tested for association with PD. Some of the CC study populations were very small, and therefore the power was low. Minor differences in dietary intake between cases and controls would not be detected by small studies. However, the cohort studies generally had a sufficient number of cases and still reported null results. Therefore if true differences do exist between affected and unaffected individuals, either exposure measurement must be more precise or population sizes need to be even larger to detect differences.

Both misdiagnosis of PD and underdiagnosis of early disease could weaken the effect measures, problems which are unavoidable without biological markers.

Exposure measurement. Nutritional exposures were assessed using the $24 \mathrm{~h}$ dietary recall, semi-quantitative food-frequency questionnaire and other types of interviews or questionnaires. More specific nutrition information is available at http://www.nal.usda.gov/fnic/foodcomp/ Most of the studies did not specifically state that the interviewers 
were blinded to CC status; therefore interviewer bias may have been introduced.

The shortest type of measurement instrument is the $24 \mathrm{~h}$ recall, which is meant to estimate current dietary intake and assumes minimal day-to-day variation. Food-frequency questionnaires have been validated and are considered acceptable approximations for dietary intake for a period of time (Willett et al. 1985). Other studies had their own structured questionnaires and the validation was often not described.

The qualitative measures, most often 'ever $v$. never' or 'yes or no', may be too simplistic to detect subtle differences between PD patients and unaffected individuals. However, in the case of alcohol and coffee intake, significant differences were detected. These exposures may be more likely than foods to have extreme amounts of consumption.

The validity of measuring nutritional intake retrospectively is often debated because it may not be representative of premorbid dietary intake. Recall bias cannot be avoided in a retrospective CC study, but results must then be interpreted with caution and verified in cohort studies. In cohort studies, the time period between exposure and outcome should be long enough for the disease to have developed after the exposure. The length of the latent period before the onset of PD symptoms is unknown, but it may be longer than 10 years.

The nutritional exposures were grouped into similar food groups and nutrients, but even within the categories there was heterogeneity. The measures are not truly comparable, although concordant results within a category may indicate an association. Foods have many components, and if an effect is found, the component responsible will need to be identified from amongst many nutrients.

The qualitative measures were often binary, but some studies classified exposure as above or below the median, or in quantiles. A larger number of quantiles would create more extreme comparisons between the highest and lowest categories, which would make it more likely to find a significant difference between the groups, assuming a reasonable population size. However, the numbers in each category would become less, which would widen the CI.

Comparisons between OR and RR reported by different studies will have to be interpreted with caution because they used different methods of measurement and calculation. Many of the studies adjusted for a number of possible confounders but most did not state how they choose the variables. The only estimate in common is a crude estimate. A solution in future studies may be to present an estimate adjusted for the minimum core confounders such as age, sex, and smoking, which have strong evidence to suggest confounding effects. Another approach would be to present stratified results, which some cohorts have done for men and women, but with small numbers this can be problematic.

Diagnostic criteria. At the moment, there are no alternatives to relying on a clinical diagnosis of PD, so it is assumed that some proportion of cases will be misdiagnosed (Hughes et al. 1992b). Both misdiagnosis of PD and underdiagnosis of early disease could weaken the effect measures, but the problems are unavoidable.
The methods of diagnosis included neurologist examination, medical record review, diagnosis logs, pharmacy databases, and telephone and mail questionnaires. Mailing questionnaires or searching records is less costly but also less comprehensive than conducting neurological examinations. Pharmacy records are a good confirmation of PD because many drugs are disease specific; however, not all patients with diagnosed PD take anti-Parkinsonian medication.

The methods of diagnosis and criteria can greatly impact which individuals are classified as affected with PD (de Rijk et al. 1997b). Neurological examination of all individuals to diagnose or exclude PD would be preferred, although in the absence of validated biomarkers (Michell et al. 2004) there would still be a possibility of misdiagnosis. Examinations are not always practical or possible; therefore medical records and databases have been utilised.

\section{Summary of results}

The majority of studies, even those testing many variables, did not find significant associations between nutritional factors and PD (summarised in Table 4). With the exception of coffee and alcohol, there were six or less of each study type, $\mathrm{CC}$ or cohort, examining each broad nutrition group. For the few nutrients and total energy intake that were found to be significantly associated with PD in CC studies, the findings were not consistently replicated in cohort studies.

The updated meta-analysis of the association between coffee drinking and PD in CC studies was similar to the past estimate (Hernan et al. 2002). There were no new cohort studies to be assessed. The meta-analyses of alcohol drinking and PD showed a significant inverse association in both the cohort and CC study pooled estimates.

\section{Conclusions and recommendations}

The aetiology of idiopathic PD probably involves both genetic and environmental factors. Few, if any, environmental exposures have been conclusively associated with PD. Nutrition is a challenging exposure to measure because of issues with recall and validation, but it is important to continue research in this area because of the possible effects on PD.

Retrospective measurement of dietary intake is problematic. Associations found in CC studies but not replicated in cohort studies are questionable. Prospective studies are necessary to support past findings and to examine new hypotheses. There were no conclusive associations between PD and any of the foods and nutrients in the present review. Coffee and alcohol intake are the only two exposures that have a relatively large number of studies in agreement that there may be inverse associations with PD. However, there are also studies with estimates in the opposite direction and null findings.

The main a priori hypotheses regarding nutritional factors and PD were not strongly supported by past study findings. The proposed protective effects of antioxidants, and conversely the harmful effects of oxidative stress, were not strongly supported by the null findings from most studies examining fruits and vegetables, vitamins, fats and Fe. The hypothesis that alcohol might cause oxidative stress 
Table 4. Summary of evidence of association between nutritional factors and Parkinson's disease (PD) from nutritional factor studies

\begin{tabular}{|c|c|c|c|c|c|c|}
\hline \multirow[b]{2}{*}{ Food or nutrient } & \multicolumn{3}{|c|}{ Case-control studies } & \multicolumn{3}{|c|}{ Cohort studies* } \\
\hline & Null & Increase & Decreaseł & Null & Increase & Decrease \\
\hline Fruit & 4 & - & - & 2 & - & - \\
\hline Vegetables & 3 & 1 & - & 2 & - & - \\
\hline Vitamin A & 2 & - & - & 2 & - & - \\
\hline Carotenes & 5 & - & - & 2 & - & - \\
\hline Lutein & 1 & 2 & - & 2 & - & - \\
\hline Lycopene & 1 & (1) & - & 2 & - & - \\
\hline Xanthophylls, xanthins & - & 1 & - & - & - & - \\
\hline Vitamin C & 5 & - & - & 3 & 1 & - \\
\hline Vitamin E & 5 & - & - & 2 & - & 1 \\
\hline Carbohydrates & 2 & 1 & - & 2 & - & - \\
\hline Fats & 3 & 2 & - & 3 & - & - \\
\hline Cholesterol & 1 & - & - & 2 & - & - \\
\hline Protein, meat & 5 & - & - & 2 & - & - \\
\hline Fish & 1 & - & 1 & 2 & - & - \\
\hline Egg & & - & 1 & - & - & - \\
\hline Dairy & 1 & - & - & 1 & 2 & - \\
\hline Alcohol (binary) & 11 & - & 2 & 3 & - & 1 \\
\hline Alcohol (quantitative) & 3 & - & 2 & 2 & - & 1 \\
\hline Beer & - & - & 2 & 2 & - & - \\
\hline Wine & 1 & - & 1 & 2 & - & - \\
\hline Liquor & - & - & 3 & 2 & - & - \\
\hline Coffee (binary) & 6 & - & 4 & 3 & - & 2 \\
\hline Coffee (quantitative) & 4 & - & 3 & 4 & - & 2 \\
\hline Tea & 3 & 1 & 4 & 1 & - & - \\
\hline Cola & 1 & - & - & - & - & - \\
\hline Caffeine & - & - & - & - & - & 1 \\
\hline Junk food & 1 & 1 & & 2 & - & - \\
\hline Legumes & - & - & - & - & - & 1 \\
\hline Nuts, seeds & - & 1 & - & 1 & - & 1 \\
\hline $\mathrm{Ca}$ & 2 & - & - & 1 & - & - \\
\hline $\mathrm{Fe}$ & 2 & (2) & - & - & - & - \\
\hline Multivitamins & 2 & - & - & 2 & - & - \\
\hline Thiamin & 1 & - & - & - & - & - \\
\hline Riboflavin & 2 & - & - & - & - & - \\
\hline Niacin & 1 & - & 1 & - & - & - \\
\hline Pantothenic acid & - & - & 1 & - & - & - \\
\hline Pyridoxine & 1 & - & 1 & 2 & - & - \\
\hline Folate & 1 & - & 1 & 2 & - & - \\
\hline Cobalamin & 1 & - & - & 2 & - & - \\
\hline Total energy & 2 & 2 & - & 2 & - & - \\
\hline $\mathrm{Cu}$ & 1 & - & - & - & - & - \\
\hline $\mathrm{Mg}$ & 1 & - & - & _- & - & - \\
\hline $\mathrm{Mn}$ & 1 & - & - & - & - & - \\
\hline $\mathrm{P}$ & 1 & - & - & - & - & - \\
\hline K & 1 & - & - & - & - & - \\
\hline $\mathrm{Se}$ & 1 & - & - & - & - & - \\
\hline $\mathrm{Na}$ & 1 & - & - & - & - & - \\
\hline Uric acid & - & - & - & 1 & - & - \\
\hline Vitamin D & 1 & - & - & 1 & - & - \\
\hline $\mathrm{Zn}$ & 1 & - & - & - & - & - \\
\hline
\end{tabular}

* Cohort and nested case-control studies.

† Increase indicates a significant positive association between factor and PD. Values in parentheses represent borderline significant results.

‡ Decrease indicates a significant inverse association between factor and PD.

was opposed by the consistent reporting of a protective association between PD and alcohol intake.

In general, a priori hypotheses about the involvement of certain nutritional factors in PD were not specified. Instead, what appeared to be the total or majority of factors from the questionnaire were included in the analysis. Future research on this topic should involve setting out a priori hypotheses regarding specific nutrients and their possible role in PD, rather than unjustified multiple testing. Many of the studies tested all available data from food-frequency questionnaires without hypotheses and even if there were some positive results, this could always be due to chance. False positive results are less likely with larger population sizes, but confirmation in multiple unrelated studies is more convincing evidence that an association is real. The methods for analysis should also be specified before viewing the data. This would involve deciding how to calculate the risk estimates.

Conducting epidemiological studies is difficult and an ideal study is usually impossible; however, the study 
methodology should be as robust as possible. The specified diagnostic criteria should be well recognised, and exclude secondary Parkinsonism. Adjustments in the analyses should be appropriate, and the steps taken to select adjustment factors should be reported. A crude association was the only estimate common across studies. An agreed minimum set of adjustments such as age, sex and smoking would be helpful for comparisons across studies with further adjustment depending on the specific model. Another option is stratification, but this is difficult to synthesise into a systematic review.

Systematic review of the literature is valuable to suggest new areas of research and to evaluate whether there is enough quality evidence to set aside hypotheses. The strongest associations were found with alcohol, caffeine and dairy products and biological research in the future will hopefully be able to elucidate their role in PD. There were a limited number of studies examining other nutrients and foods; therefore more evidence is needed to decide whether to pursue research in those areas.

Areas of future interest include studying the interaction of diet and PD with other factors. The gene-diet interaction (Mattson, 2003) has been explored in animals and needs to be confirmed in human subjects. Studying the effects of dietary patterns on disease outcomes may reveal nutrient interactions. The interaction between nutrition, vascular disease, and PD is interesting because nutritional factors have been implicated in both diseases, and vascular disease is a cause of at least one type of Parkinsonism (Foltynie et al. 2002). Large cohort studies may be able to take the approach that has been used in dementia and nutrition studies, and use blood biomarkers prospectively, but large numbers will be necessary.

\section{Conflict of interest}

L. I.'s studentship is funded by GlaxoSmithKline Pharmaceuticals. However, they have had no involvement or input into the present article.

\section{Acknowledgements}

The authors would like to thank Anna Marnik and Cynthia Ishihara for obtaining references; Mei Chan, Ian Cooper, Jane Fleming, Masako Kataoka, Nadezda Novakovich, Philip Paul, Michelle To, Kohei Watanabe, and Julia Zaccai for translating foreign articles; Sheila Bingham, Ailsa Welch, and Ken Ishihara for reading and commenting on earlier drafts; Professor Kay-Tee Khaw for commentary on literature quality assessment; and Christ's College Cambridge.

\section{References}

Aarsland D, Andersen K, Larsen JP, Lolk A \& Kragh-Sorensen P (2003) Prevalence and characteristics of dementia in Parkinson disease: an 8-year prospective study. Archives of Neurology $\mathbf{6 0}$, 387-392.

Ahmadi A, Fredrikson M, Jerregard H, Akerback A, Fall PA, Rannug A, Axelson O \& Soderkvist P (2000) GSTM1 and mEPHX polymorphisms in Parkinson's disease and age of onset.
Biochemical and Biophysical Research Communications 269, 676-680.

Ames BN, Cathcart R, Schwiers E \& Hochstein P (1981) Uric acid provides an antioxidant defense in humans against oxidant- and radical-caused aging and cancer: a hypothesis. Proceedings of the National Academy of Science U S A 78, 6858-6862.

Anderson C, Checkoway H, Franklin GM, Beresford S, SmithWeller T \& Swanson PD (1999) Dietary factors in Parkinson's disease: the role of food groups and specific foods. Movement Disorders 14, 21-27.

Ascherio A, Zhang SM, Hernan MA, Kawachi I, Colditz GA, Speizer FE \& Willett WC (2001) Prospective study of caffeine consumption and risk of Parkinson's disease in men and women. Annals of Neurology 50, 56-63.

Behari M, Srivastava AK, Das RR \& Pandey RM (2001) Risk factors of Parkinson's disease in Indian patients. Journal of Neurological Science 190, 49-55.

Ben-Shlomo Y (1998) Chapter 1. In The Epidemiology of Neurological Disorders, pp. 1-33 [C Martyn and R Hughes, editors]. London: BMJ Books.

Bender DA (1989) Vitamin B6 requirements and recommendations. European Journal of Clinical Nutrition 43, 289-309.

Benedetti MD, Bower JH, Maraganore DM, McDonnell SK, Peterson BJ, Ahlskog JE, Schaid DJ \& Rocca WA (2000) Smoking, alcohol, and coffee consumption preceding Parkinson's disease: a case-control study. Neurology 55, 1350-1358.

Berg D, Gerlach M, Youdim MB, Double KL, Zecca L, Riederer P \& Becker G (2001) Brain iron pathways and their relevance to Parkinson's disease. Journal of Neurochemistry 79, 225-236.

Bharath S, Hsu M, Kaur D, Rajagopalan S \& Andersen JK (2002) Glutathione, iron and Parkinson's disease. Biochemical Pharmacology 64, 1037-1048.

Butterfield DA, Castegna A, Drake J, Scapagnini G \& Calabrese V (2002) Vitamin E and neurodegenerative disorders associated with oxidative stress. Nutritional Neuroscience 5, 229-239.

Butterfield PG, Valanis BG, Spencer PS, Lindeman CA \& Nutt JG (1993) Environmental antecedents of young-onset Parkinson's disease. Neurology 43, 1150-1158.

Calne DB, Eisen A, McGeer E \& Spencer P (1986) Alzheimer's disease, Parkinson's disease, and motoneurone disease: abiotrophic interaction between ageing and environment? Lancet ii, 1067-1070.

Cerhan JR, Wallace RB \& Folsom AR (1994) Antioxidant intake and the risk of Parkinson's disease (PD) in older women. American Journal of Epidemiology 139, S65.

Chan DK, Woo J, Ho SC, Pang CP, Law LK, Ng PW, Hung WT, Kwok T, Hui E, Orr K, Leung MF \& Kay R (1998) Genetic and environmental risk factors for Parkinson's disease in a Chinese population. Journal of Neurology, Neurosurgery and Psychiatry 65, 781-784.

Checkoway H, Powers K, Smith-Weller T, Franklin GM, Longstreth WT Jr \& Swanson PD (2002) Parkinson's disease risks associated with cigarette smoking, alcohol consumption, and caffeine intake. American Journal of Epidemiology 155, $732-738$.

Chen H, Zhang SM, Hernan MA, Willett WC \& Ascherio A (2002) Diet and Parkinson's disease: a potential role of dairy products in men. Annals of Neurology 52, 793-801.

Chen H, Zhang SM, Hernan MA, Willett WC \& Ascherio A (2003) Dietary intakes of fat and risk of Parkinson's disease. American Journal of Epidemiology 157, 1007-1014.

Chen H, Zhang SM, Schwarzschild MA, Hernan MA, Logroscino G, Willett WC \& Ascherio A (2004) Folate intake and risk of Parkinson's disease. American Journal of Epidemiology 160, $368-375$. 
Church WH \& Ward VL (1994) Uric acid is reduced in the substantia nigra in Parkinson's disease: effect on dopamine oxidation. Brain Research Bulletin 33, 419-425.

Cleland LG, James MJ \& Proudman SM (2003) The role of fish oils in the treatment of rheumatoid arthritis. Drugs 63, 845-853.

Collins MA (2002) Alkaloids, alcohol and Parkinson's disease. Parkinsonism and Related Disorders 8, 417-422.

Combs GF Jr \& Gray WP (1998) Chemopreventive agents: selenium. Pharmacology and Therapeutics 79, 179-192.

Cox PA \& Sacks OW (2002) Cycad neurotoxins, consumption of flying foxes, and ALS-PDC disease in Guam. Neurology 58, 956-959.

Davies KN, King D \& Davies H (1994) A study of the nutritional status of elderly patients with Parkinson's disease. Age and Ageing 23, 142-145.

Davis JW, Grandinetti A, Waslien CI, Ross GW, White LR \& Morens DM (1996) Observations on serum uric acid levels and the risk of idiopathic Parkinson's disease. American Journal of Epidemiology 144, 480-484.

de Rijk MC, Breteler MM, den Breeijen JH, Launer LJ, Grobbee DE, van der Meche FG \& Hofman A (1997a) Dietary antioxidants and Parkinson disease. The Rotterdam Study. Archives of Neurology 54, 762-765.

de Rijk MC, Rocca WA, Anderson DW, Melcon MO, Breteler MM \& Maraganore DM (1997b) A population perspective on diagnostic criteria for Parkinson's disease. Neurology 48, $1277-1281$

de Rijk MC, Tzourio C, Breteler MM, Dartigues JF, Amaducci L, Lopez-Pousa S, Manubens-Bertran JM, Alperovitch A \& Rocca WA (1997c) Prevalence of Parkinsonism and Parkinson's disease in Europe: the EUROPARKINSON Collaborative Study. European Community Concerted Action on the Epidemiology of Parkinson's disease. Journal of Neurology, Neurosurgery and Psychiatry 62, 10-15.

de Silva HR, Khan NL \& Wood NW (2000c) The genetics of Parkinson's disease. Current Opinion in Genetics and Development 10, 292-298.

Desagher S, Glowinski J \& Premont J (1996) Astrocytes protect neurons from hydrogen peroxide toxicity. Journal of Neuroscience 16, 2553-2562.

Ensminger A (1994) Foods and Nutrition Encyclopedia. London and Boca Raton: CRC Press.

Etminan M, Gill SS \& Samii A (2005) Intake of vitamin E, vitamin $\mathrm{C}$, and carotenoids and the risk of Parkinson's disease: a metaanalysis. Lancet Neurology 4, 362-365.

Fall PA, Fredrikson M, Axelson O \& Granerus AK (1999) Nutritional and occupational factors influencing the risk of Parkinson's disease: a case-control study in southeastern Sweden. Movement Disorders 14, 28-37.

Fariss MW \& Zhang JG (2003) Vitamin E therapy in Parkinson's disease. Toxicology 189, 129-146.

Fink JS, Bains LA, Beiser A, Seshadri S \& Wolf PA (2001) Caffeine intake and the risk of incident Parkinson's disease: the Framingham study. Movement Disorders 16, 984.

Foley P \& Riederer P (2000) Influence of neurotoxins and oxidative stress on the onset and progression of Parkinson's disease. Journal of Neurology 247, Suppl. 2, II82-II94.

Folstein MF, Folstein SE \& McHugh PR (1975) 'Mini-mental state'. A practical method for grading the cognitive state of patients for the clinician. Journal of Psychiatric Research 12, 189-198.

Foltynie T, Barker R \& Brayne C (2002) Vascular Parkinsonism: a review of the precision and frequency of the diagnosis. Neuroepidemiology 21, 1-7.

Fredholm BB, Battig K, Holmen J, Nehlig A \& Zvartau EE (1999) Actions of caffeine in the brain with special reference to factors that contribute to its widespread use. Pharmacological Reviews 51, 83-133.

Glasziou P, Irwig L, Bain C \& Colditz G (2001) Systematic Reviews in Healthcare: a Practical Guide. Cambridge, UK: Cambridge University Press.

Gonthier B, Signorini-Allibe N, Soubeyran A, Eysseric H, Lamarche F \& Barret L (2004) Ethanol can modify the effects of certain free radical-generating systems on astrocytes. Alcoholism, Clinical and Experimental Research 28, 526-534.

Gorell JM, Johnson CC, Rybicki BA, Peterson EL, Kortsha GX, Brown GG \& Richardson RJ (1997) Occupational exposures to metals as risk factors for Parkinson's disease. Neurology 48, $650-658$.

Gorell JM, Rybicki BA, Johnson CC \& Peterson EL (1999) Smoking and Parkinson's disease: a dose-response relationship. Neurology 52, 115-119.

Grandinetti A, Morens DM, Reed D \& MacEachern D (1994) Prospective study of cigarette smoking and the risk of developing idiopathic Parkinson's disease. American Journal of Epidemiology 139, 1129-1138.

Gwinn-Hardy K (2002) Genetics of Parkinsonism. Movement Disorders 17, 645-656.

Haack DG, Baumann RJ, McKean HE, Jameson HD \& Turbek JA (1981) Nicotine exposure and Parkinson disease. American Journal of Epidemiology 114, 191-200.

Halliwell B (1992) Reactive oxygen species and the central nervous system. Journal of Neurochemistry 59, 1609-1623.

Hellenbrand W, Boeing H, Robra BP, Seidler A, Vieregge P, Nischan P, Joerg J, Oertel WH, Schneider E \& Ulm G (1996a) Diet and Parkinson's disease. II: A possible role for the past intake of specific nutrients. Results from a self-administered food-frequency questionnaire in a case-control study. Neurology 47, 644-650.

Hellenbrand W, Seidler A, Boeing H, Robra BP, Vieregge P, Nischan P, Joerg J, Oertel WH, Schneider E \& Ulm G (1996b) Diet and Parkinson's disease. I: A possible role for the past intake of specific foods and food groups. Results from a selfadministered food-frequency questionnaire in a case-control study. Neurology 47, 636-643.

Herbert V (1996) Vitamin B12 in Present Knowledge in Nutrition. Washington, DC: International Life Sciences Institute Press.

Herbert V (1999) Folic acid. In Modern Nutrition in Health and Disease, pp. 433-446 [M Shils, J Olson, M Shike and AC Ross, editors]. Baltimore, MA: Williams \& Wilkins.

Hernan MA, Chen H, Schwarzschild MA \& Ascherio A (2003) Alcohol consumption and the incidence of Parkinson's disease. Annals of Neurology 54, 170-175.

Hernan MA, Takkouche B, Caamano-Isorna F \& Gestal-Otero JJ (2002) A meta-analysis of coffee drinking, cigarette smoking, and the risk of Parkinson's disease. Annals of Neurology 52, 276-284.

Ho SC, Woo J \& Lee CM (1989) Epidemiologic study of Parkinson's disease in Hong Kong. Neurology 39, 1314-1318.

Hughes AJ, Ben Shlomo Y, Daniel SE \& Lees AJ (1992a) What features improve the accuracy of clinical diagnosis in Parkinson's disease: a clinicopathologic study. Neurology 42, $1142-1146$

Hughes AJ, Daniel SE, Kilford L \& Lees AJ (1992b) Accuracy of clinical diagnosis of idiopathic Parkinson's disease: a clinicopathological study of 100 cases. Journal of Neurology, Neurosurgery and Psychiatry 55, 181-184.

Institute of Medicine (1999) Dietary Reference Intakes: Calcium, Phosphorus, Magnesium, Vitamin D and Fluoride. Washington, DC: National Academy Press.

Institute of Medicine (2001) Dietary Reference Intakes: Vitamin A, Vitamin K, Arsenic, Boron, Chromium, Copper, Iodine, Iron, 
Manganese, Molybdenum, Nickel, Silicon, Vanadium, and Zinc. Washington, DC: National Academy Press.

Jenner P (2003) Oxidative stress in Parkinson's disease. Annals of Neurology 53, Suppl. 3, S26-S36.

Jimenez-Jimenez FJ, Mateo D \& Gimenez-Roldan S (1992) Premorbid smoking, alcohol consumption, and coffee drinking habits in Parkinson's disease: a case-control study. Movement Disorders 7, 339-344.

Johnson CC, Gorell JM, Rybicki BA, Sanders K \& Peterson EL (1999) Adult nutrient intake as a risk factor for Parkinson's disease. International Journal of Epidemiology 28, 1102-1109.

Khan KS (2003) Systematic Reviews to Support Evidence-Based Medicine: How to Review and Apply Findings of Healthcare Research. London: The Royal Society of Medicine Press.

Koutsilieri E, Scheller C, Grunblatt E, Nara K, Li J \& Riederer P (2002) Free radicals in Parkinson's disease. Journal of Neurology 249, Suppl. 2, II1-II5.

Lang AE \& Lozano AM (1998) Parkinson's disease. First of two parts. New England Journal of Medicine 339, 1044-1053.

Langston JW, Widner H, Goetz CG, Brooks D, Fahn S, Freeman T \& Watts R (1992) Core assessment program for intracerebral transplantations (CAPIT). Movement Disorders 7, 2-13.

Liddle J (1996) Method for Evaluating Research and Guideline Evidence. Sydney: New South Wales Department of Health.

Liou HH, Tsai MC, Chen CJ, Jeng JS, Chang YC, Chen SY \& Chen RC (1997) Environmental risk factors and Parkinson's disease: a case-control study in Taiwan. Neurology 48, 1583-1588.

Logroscino G, Marder K, Cote L, Tang MX, Shea S \& Mayeux R (1996) Dietary lipids and antioxidants in Parkinson's disease: a population-based, case-control study. Annals of Neurology 39, 89-94.

Logroscino G, Marder K, Graziano J, Freyer G, Slavkovich V, Lojacono N, Cote L \& Mayeux R (1998) Dietary iron, animal fats and risk of Parkinson's disease. Movement Disorders 13, Suppl. 1, S13-S16.

Maraganore DM, Harding AE \& Marsden CD (1991) A clinical and genetic study of familial Parkinson's disease. Movement Disorders 6, 205-211.

Mattson MP (2003) Gene-diet interactions in brain aging and neurodegenerative disorders. Annals of Internal Medicine 139, 441-444.

Mayeux R, Tang MX, Marder K, Cote LJ \& Stern Y (1994) Smoking and Parkinson's disease. Movement Disorders 9, 207-212.

Michell AW, Lewis SJ, Foltynie T \& Barker RA (2004) Biomarkers and Parkinson's disease. Brain 127, 1693-1705.

Miller JW (2002) Homocysteine, folate deficiency, and Parkinson's disease. Nutrition Reviews 60, 410-413.

Morano A, Jimenez-Jimenez FJ, Molina JA \& Antolin MA (1994) Risk-factors for Parkinson's disease: case-control study in the province of Caceres. Spain. Acta Neurologica Scandinavica 89, $164-170$.

Morens DM, Grandinetti A, Waslien CI, Park CB, Ross GW \& White LR (1996) Case-control study of idiopathic Parkinson's disease and dietary vitamin E intake. Neurology 46, 1270-1274.

Morris MS (2002) Folate, homocysteine, and neurological function. Nutrition in Clinical Care 5, 124-132.

Nefzger MD, Quadfasel FA \& Karl VC (1968) A retrospective study of smoking in Parkinson's disease. American Journal of Epidemiology 88, 149-158.

Nelson LM, Van den Eeden S, Tanner CM, Bernstein A \& Harrington D (1999) Association of alcohol and tobacco consumption with Parkinson's disease: a population-based study. Neurology 52, A538-A539.

Oxman AD (1994) Checklists for review articles. BMJ 309, 648-651.
Paganini-Hill A (2001) Risk factors for Parkinson's disease: the leisure world cohort study. Neuroepidemiology 20, 118-124.

Park M, Ross GW, Petrovitch H, White LR, Masaki KH, Nelson JS, Tanner CM, Curb JD, Blanchette PL \& Abbott RD (2005) Consumption of milk and calcium in midlife and the future risk of Parkinson disease. Neurology 64, 1047-1051.

Parkinson J (1817) An essay on the shaking palsy. London: Sherwood, Neely \& Jones.

Pollock BG, Wylie M, Stack JA, Sorisio DA, Thompson DS, Kirshner MA, Folan MM \& Condifer KA (1999) Inhibition of caffeine metabolism by estrogen replacement therapy in postmenopausal women. Journal of Clinical Pharmacology 39, 936-940.

Powers KM, Smith-Weller T, Franklin GM, Longstreth WT Jr, Swanson PD \& Checkoway H (2003) Parkinson's disease risks associated with dietary iron, manganese, and other nutrient intakes. Neurology 60, 1761-1766.

Preux PM, Condet A, Anglade C, Druet-Cabanac M, Debrock C, Macharia W, Couratier P, Boutros-Toni F \& Dumas M (2000) Parkinson's disease and environmental factors. Matched casecontrol study in the Limousin region, France. Neuroepidemiology 19, 333-337.

Ragonese P, Salemi G, Morgante L, Aridon P, Epifanio A, Buffa D, Scoppa F \& Savettieria G (2003) A case-control study on cigarette, alcohol, and coffee consumption preceding Parkinsons disease. Neuroepidemiology 22, 297-304.

Rao AV \& Balachandran B (2002) Role of oxidative stress and antioxidants in neurodegenerative diseases. Nutritional Neuroscience 5, 291-309.

Ross GW, Abbott RD, Petrovitch H, Morens DM, Grandinetti A, Tung KH, Tanner CM, Masaki KH, Blanchette PL, Curb JD, Popper JS \& White LR (2000) Association of coffee and caffeine intake with the risk of Parkinson disease. Journal of the American Medical Association 283, 2674-2679.

Salem N Jr, Litman B, Kim HY \& Gawrisch K (2001) Mechanisms of action of docosahexaenoic acid in the nervous system. Lipids 36, 945-959.

Scheider WL, Hershey LA, Vena JE, Holmlund T, Marshall JR \& Freudenheim JL (1997) Dietary antioxidants and other dietary factors in the etiology of Parkinson's disease. Movement Disorders 12, 190-196.

Scottish Intercollegiate Guidelines Network (2002) SIGN 50: a guideline developers' handbook. SIGN publication no. 50. Accessed 24 October 2005. http://www.sign.ac.uk/guidelines/ fulltext/50/

Smargiassi A, Mutti A, De Rosa A, De Palma G, Negrotti A \& Calzetti S (1998) A case-control study of occupational and environmental risk factors for Parkinson's disease in the EmiliaRomagna region of Italy. Neurotoxicology 19, 709-712.

Tan EK, Tan C, Fook-Chong SM, Lum SY, Chai A, Chung H, Shen H, Zhao Y, Teoh ML, Yih Y, Pavanni R, Chandran VR \& Wong MC (2003) Dose-dependent protective effect of coffee, tea, and smoking in Parkinson's disease: a study in ethnic Chinese. Journal of Neurological Science 216, 163-167.

Tanner CM (2003) Is the cause of Parkinson's disease environmental or hereditary? Evidence from twin studies. Advances in Neurology 91, 133-142.

Tanner CM \& Ben Shlomo Y (1999) Epidemiology of Parkinson's disease. Advances in Neurology 80, 153-159.

Taylor CA, Saint-Hilaire MH, Cupples LA, Thomas CA, Burchard AE, Feldman RG \& Myers RH (1999) Environmental, medical, and family history risk factors for Parkinson's disease: a New England-based case control study. American Journal of Medical Genetics 88, 742-749.

Tohgi H, Abe T, Takahashi S \& Kikuchi T (1993) The urate and xanthine concentrations in the cerebrospinal fluid in patients with vascular dementia of the Binswanger type, Alzheimer type 
dementia, and Parkinson's disease. Journal of Neural Transmission. Parkinson's Disease and Dementia Section 6, 119-126.

Tsai CH, Lo SK, See LC, Chen HZ, Chen RS, Weng YH, Chang FC \& Lu CS (2002) Environmental risk factors of young onset Parkinson's disease: a case-control study. Clinical Neurology and Neurosurgery 104, 328-333.

US Department of Agriculture (2004) USDA Nutrient Database for Standard Reference, release 15, Nutrient Data Laboratory home page. Accessed 24 October 2005. http://www.nal.usda.gov/fnic/ foodcomp

Verhoef P, Stampfer MJ, Buring JE, Gaziano JM, Allen RH, Stabler SP, Reynolds RD, Kok FJ, Hennekens CH \& Willett WC (1996) Homocysteine metabolism and risk of myocardial infarction: relation with vitamins B6, B12, and folate. American Journal of Epidemiology 143, 845-859.

Wang WZ, Fang XH, Cheng XM, Jiang DH \& Lin ZJ (1993) A casecontrol study on the environmental risk factors of Parkinson's disease in Tianjin, China. Neuroepidemiology 12, 209-218.

Ward CD \& Gibb WR (1990) Research diagnostic criteria for Parkinson's disease. Advances in Neurology 53, 245-249.

Weiner WJ \& Lang AE (1989) Movement Disorders: a Comprehensive Survey, pp. 23-115. New York: Futura.

Weinreb O, Mandel S, Amit T \& Youdim MB (2004) Neurological mechanisms of green tea polyphenols in Alzheimer's and Parkinson's diseases. Journal of Nutritional Biochemistry 15, 506-516.

Wells GA, Shea B, Peterson J, Welch V, Losos M and Tugwell P (2003) The Newcastle-Ottawa Scale (NOS) for assessing the quality of nonrandomised studies in meta-analyses. Accessed 24 October 2005. http://www.lri.ca/programs/ceu/oxford.htm

Willems-Giesbergen P, de Rijk MC, van Swieten JC \& Hofman A (2000) Smoking, alcohol, and coffee consumption and the risk of PD: results from the Rotterdam study. Neurology 54, A347-A348.

Willett WC, Sampson L, Stampfer MJ, Rosner B, Bain C, Witschi J, Hennekens CH \& Speizer FE (1985) Reproducibility and validity of a semiquantitative food frequency questionnaire. American Journal of Epidemiology 122, 51-65.

Yang J, Wu Z \& Lou X (1994) A case-control study on risk factors in etiology of Parkinson's disease (article in Chinese). Zhonghua Liuxingbingxue Zazhi 15, 6-9.

Zayed J, Ducic S, Campanella G, Panisset JC, Andre P, Masson H \& Roy M (1990) Environmental factors in the etiology of Parkinson's disease (article in French). Canadian Journal of Neurological Science 17, 286-291.

Zhang SM, Hernan MA, Chen H, Spiegelman D, Willett WC \& Ascherio A (2002) Intakes of vitamins E and C, carotenoids, vitamin supplements, and PD risk. Neurology 59, 1161-1169.

Zhang ZX \& Roman GC (1993) Worldwide occurrence of Parkinson's disease: an updated review. Neuroepidemiology 12, 195-208.

\section{Appendix 1}

\section{United Kingdom Parkinson's Disease Society Brain Bank} Criteria (Hughes et al. 1992a)

Step 1 - Diagnosis of Parkinsonian syndrome:

Bradykinesia (slowness of initiation of voluntary movement with progressive reduction in speed and amplitude of repetitive actions) and at least one of the following;

Muscular rigidity;

$4-6 \mathrm{~Hz}$ rest tremor;

Postural instability not caused by primary visual, vestibular, cerebellar, or proprioceptive dysfunction.

Step 2 - Exclusion criteria for Parkinson's disease:

History of repeated strokes with stepwise progression of Parkinsonian features;

History of repeated head injury;

History of definite encephalitis;

Oculogyric crises;

Neuroleptic treatment at onset of symptoms;

More than one affected relative;

Sustained remission;

Strictly unilateral features after 3 years;

Supranuclear gaze palsy;

Cerebellar signs;

Early severe autonomic involvement;

Early severe dementia with disturbances of memory, language, and praxis;

Babinski sign;

Presence of cerebral tumour or communicating hydrocephalus on computed tomography scan;

Negative response to large doses of levodopa (if malabsorption excluded);

1-methyl-4-phenyl-1,2,3,6-tetrahydropyridine exposure.

Step 3 - Supportive prospective criteria (three or more required for diagnosis of definite Parkinson's disease):

Unilateral onset;

Rest tremor present;

Progressive disorder;

Persistent asymmetry affecting side of onset most;

Excellent response (70-100\%) to levodopa;

Severe levodopa-induced chorea;

Levodopa response for 5 years or more;

Clinical course of 10 years or more. 